\title{
Comparative tolerability of targeted therapies in pulmonary hypertension
}

Magdalena Jasińska-Stroschein, Karolina Stawarczyk, Anna Stępień, Daria Orszulak-Michalak

Department of Biopharmacy, Medical University of Lodz, Lodz, Poland

Submitted: 10 March 2019

Accepted: 11 November 2019

Arch Med Sci

DOI: https://doi.org/10.5114/aoms.2020.96143

Copyright @ 2020 Termedia \& Banach

\section{Abstract}

Introduction: The objective of this study was to estimate the safety profile of pulmonary hypertension-specific therapies using placebo-controlled and active comparator trials.

Material and methods: The search corpus comprised Medline, Scopus, Embase and Clinical Trials databases. A systematic review and meta-analysis was performed to assess the relative risk of severe events and discontinuations as well as of adverse drug reactions (ADRs) classified into 26 categories and 21 subcategories defined by the Medical Dictionary for Regulatory Activities (MedRA).

Results: Pulmonary hypertension-specific therapies had the greatest effect on such events as flushing and headache as well as jaw pain, limb pain and myalgia or gastrointestinal disorders. The relative risk for ADRs in patients receiving monotherapy (vs. placebo/supportive therapies) and combined regimens (vs. monotherapy) was significantly increased. The risk of cessation for the combined regimen was slightly higher $\left(\mathrm{Q}_{\text {inter-group }}, p=0.0778\right)$. Such ADRs as blood and lymphatic system disorders with the anemia subgroup, gastrointestinal disorders with diarrhea and nausea subgroups, respiratory and thoracic diseases or nervous system disorders with headache tended to occur more often in combination regimens as compared to monotherapy. Conclusions: About half of the main categories and subcategories of adverse reactions according to MedRA were associated with a relatively high frequency and hazard ratio. Their risk can be increased when combination regimens are used, especially.

Key words: pulmonary arterial hypertension, targeted drugs, adverse drug reactions, meta-analysis.

\section{Introduction}

Pulmonary hypertension $(\mathrm{PH})$ is a progressive disease of multifactorial etiology with poor prognosis due to right heart failure. It is defined by a resting mean pulmonary artery pressure (PAP) of $25 \mathrm{mmHg}$ or more. According to pathophysiological appearance the following subgroups of $\mathrm{PH}$ have been specified: primary pulmonary hypertension, called idiopathic or IPAH (idiopathic pulmonary arterial hypertension), and secondary pulmonary hypertension. The latter can be further divided into: passive - in the course of left, congestive heart failure, active - due to diseases characterized by hypoxia, poisoning, or in the case of vasoactive drug use, hyperkinetic - due to congenital heart defects with leakage from left to right heart, obstructive - in the course of collagenases and

\author{
Corresponding author: \\ Magdalena Jasińska- \\ Stroschein \\ Department of Biopharmacy \\ Medical University of Lodz \\ 1 Muszyńskiego St \\ 90-151 Lodz, Poland \\ e-mail: magdalena.jasinska- \\ stroschein@umed.lodz.pl
}


other disorders causing pressure on pulmonary capillaries, and in pulmonary embolism. Anatomopathologically, $\mathrm{PH}$ is characterized to be precapillary - arterial, post-capillary - venous or mixed.

The introduction of disease targeted therapies has significantly improved management and patient survival in the above-mentioned cases. Current guidelines propose use of two or more classes of drugs that may be applied sequentially or initially (upfront). Several randomized clinical trials and meta-analyses have revealed the efficacy of such $\mathrm{PH}$-specific therapies toward reduction of clinical worsening, defined as a combination of death, admission to hospital, lung transplantation, symptomatic progression or treatment escalation including initiation of prostacyclins [1-6].

Adverse drug reactions (ADRs) with pulmonary vasodilator use in $\mathrm{PH}$ patients are usually reported in the Summaries of Product Characteristics (SmPCs) as common (i.e. 1/10-1/100 patients) or very common (i.e. $\geq 1 / 10$ patients). They may contribute to a worse quality of life, prevent therapeutic escalation or precipitate discontinuation. In such cases, drug avoidance may in turn contribute to a worse prognosis. On the other hand, the presence of dose-related ADRs may suggest a higher circulating dose and, in some cases, be associated with improved mortality despite the impact on health-related quality of life [7].

It remains unknown whether the increased efficacy of such combinations of two or more agents targeting $\mathrm{PH}$ is accompanied by diminished safety. While there is a need to identify the efficacy-to-safety ratio of therapeutic strategies including combined regimens, there is no comprehensive analysis on the adverse event profiles of different targeted therapies in $\mathrm{PH}$.

The objective of this study was to systematically review the safety of various disease-specific agents approved to manage $\mathrm{PH}$, according to data reported in randomized, controlled clinical trials using both placebo-controlled and active comparators. We propose a comparative appraisal across particular medication classes, i.e.: endothelin receptor antagonist (ERA), prostacyclin analogues $\left(\mathrm{PGI}_{2}\right)$, prostacyclin receptor (IP) agonists, phosphodiesterase type 5 inhibitors (PDE-5i) or guanylate cyclase stimulators (GCS). Besides the therapeutic group, other determinants, such as the amount of agents added to baseline therapy (i.e. 1, 2 or more) or route of administration, were taken into consideration.

\section{Material and methods}

\section{Data source}

Meta-analysis was reported in accordance with the PRISMA guidelines [8]. The search corpus com- prised Medline, Scopus, Embase and Clinical Trials from January $1^{\text {st }} 1990$ to May $17^{\text {th }} 2018$. The databases were searched with no language restrictions using the following search terms in titles and abstracts: ('bosentan', OR 'ambrisentan', OR 'epoprostenol', OR 'treprostinil', OR 'iloprost', OR 'selexipag', OR 'sildenafil', OR 'tadalafil', OR 'riociguat', OR 'macitentan') AND 'humans', AND 'pulmonary hypertension', AND 'clinical trials'. Two investigators evaluated each article independently. The selection of abstracts was independently carried out by two different researchers (K.S. and A.S.), and disagreements were resolved through discussion with a third researcher (M.J-S.). Next, the eligibility of selected papers/trials was confirmed after reading the complete text. Each article underwent independent, blinded, double-data extraction by two reviewers, discrepancies in data extraction underwent arbitration by a third reviewer and consensus was obtained by verbal discussion.

\section{Study selection}

Inclusion criteria were defined a priori. Original studies were included if they met the following criteria: (i) being a clinical prospective, double-blind, randomized trial assessing the effects of additional $\mathrm{PH}$-targeted therapy with any dose compared with background (placebo, non-specific or specific) therapy in adult patients with $\mathrm{PH}$, (ii) recruiting patients with a clinical diagnosis of $\mathrm{PH}$ (idiopathic - IPAH, connective tissue disease - CTD, due to human immunodeficiency virus - HIV infection or drug-induced pulmonary arterial hypertension DPAH) as well as patients developing the venous form of pulmonary hypertension, e.g. due to congenital heart disease (CHD). In the placebo arm, only supportive therapy was considered: oral anticoagulants, diuretics, oxygen or digoxin and other cardiovascular drugs. The specific $\mathrm{PH}$ agents had to be withdrawn at least three months before enrolment.

Studies were excluded if any of the following criteria were met: 1 . Lack of original data; 2 . Lack of blinding; 3. Lack of control group (comparator); 4. No adverse reactions were reported/described by authors; 5 . Animal studies.

\section{Quality assessment}

The information of methodological quality was extracted as well, in particular data on random sequence generation, blinding or indications of incomplete outcome information according to Jadad criteria (0-5 pts) [9]. The quality was assessed independently by two investigators. In case of any discrepancies a discussion was carried out to achieve a consensus. 
The outcomes involved: (i) severe adverse drug reactions (SADRs), (ii) discontinuations because of ADR and (iii) particular adverse reactions that were classified according to the Medical Dictionary for Regulatory Activities (MedRA) (version 16). A structured form developed in MS Excel was used to extract data on trial and patient population characteristics and outcomes.

\section{Statistical analysis}

The meta-analysis was conducted using STATISTICA 13.1 software and Module for Meta-analysis (StatSoft, Poland). The difference of dichotomous data between two groups was estimated with relative risk (RR) with $95 \%$ two-tailed confidence intervals $(\mathrm{Cl})$. A DerSimonian and Laird random-effects model was used to compensate for the heterogeneity of studies [10]. We analyzed whether the risk of particular ADR increased when a $\mathrm{PH}$-specific agent belonging to the following groups was added to the baseline (placebo or other PH targeted agent): ERA, prostacyclin and their analogues $\left(\mathrm{PGI}_{2}\right)$, IP agonists, PDE-5i and soluble $G C s$. These findings were reported in terms of "RR parameter". In order to examine the robustness of the results, sensitivity analysis was conducted using the leave-one-out method, i.e. removing one study each time and recalculating the results.

\section{Subgroup analyses}

Heterogeneity was quantitatively assessed using Cochran's $Q$, and 12 statistics. $P<0.05$ was regarded as statistically significant. To explore the potential sources of heterogeneity, we performed particular subgroup analyses to determine the impact of route of administration (p.o., i.v., s.c., inh) and amount of agents added to baseline therapy (i.e. 1, 2 and more; monotherapy vs. combination) on the obtained outcome. Following this, all statistically significant ADRs were taken together and their risk calculated separately for monotherapy and combined regimens to determine whether combining two or more agents can significantly alter relative risk. The differences among compared factors were confirmed with a statistically significant measure of $\mathrm{Q}_{\text {inter-group }}$ value $(p<0.05)$.

Potential publication bias was examined using a visual inspection of Begg's funnel plot asymmetry, Begg's rank correlation, and Egger's weighted regression [11, 12]. Duval and Tweedie 'trim and fill' was used to adjust the analysis for the effects of publication bias [13].

\section{Results}

\section{Characteristics of included trials}

In total, 1701 articles were screened; 449 duplicates were removed, and another 837 were ex- cluded because they did not meet inclusion criteria. Out of 415 eligible papers, 380 were excluded since they were not blinded $(n=168)$; they did not have comparator $(n=91)$; or the adverse events were not mentioned by the authors $(n=80)$. Other reasons included e.g. lack of original data, only non-specific or non-pharmacological therapy that was introduced for pulmonary hypertension. Finally, we included 35 articles in this meta-analysis (Figure 1). In one study (acronym: AMBITION) two arms were included separately, and 36 trials were analyzed, finally. The median Jadad score was three out of five (interquartile range [IQR]: 3-4) (Table I).

All the analyzed studies were randomized, double-blinded and placebo controlled. This meta-analysis comprised $7977 \mathrm{PH}$ patients: 4674 with $\mathrm{iPAH}$, 2082 with CTD-PAH, 595 with pulmonary hypertension due to CHD, 112 with DPAH, and 514 with other forms of $\mathrm{PH}$, including idiopathic pulmonary fibrosis, sickle cell disease, portopulmonary hypertension or $\mathrm{PH}$ associated with atrial septal defect.

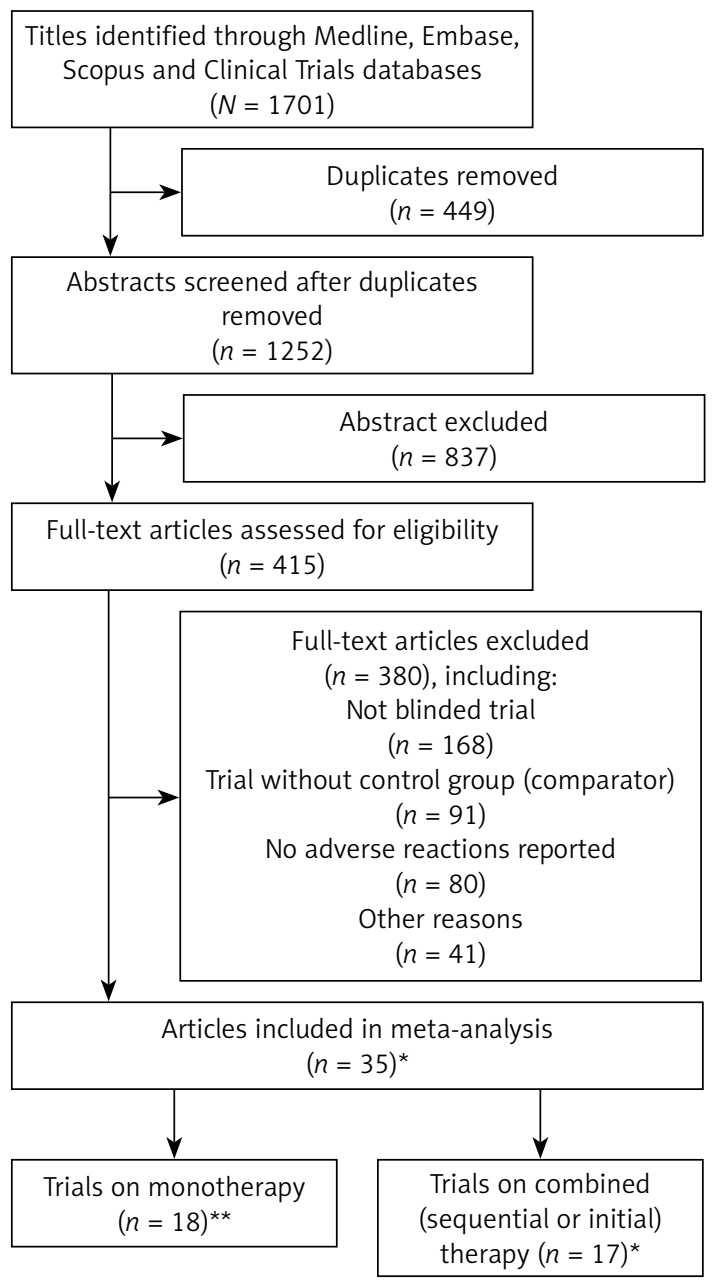

*In one study: two arms were included separately (AMBITION). **In one article the results of two trials were reported (ARIES-1 and ARIES-2).

Figure 1. Flow diagram of trial identification and selection 


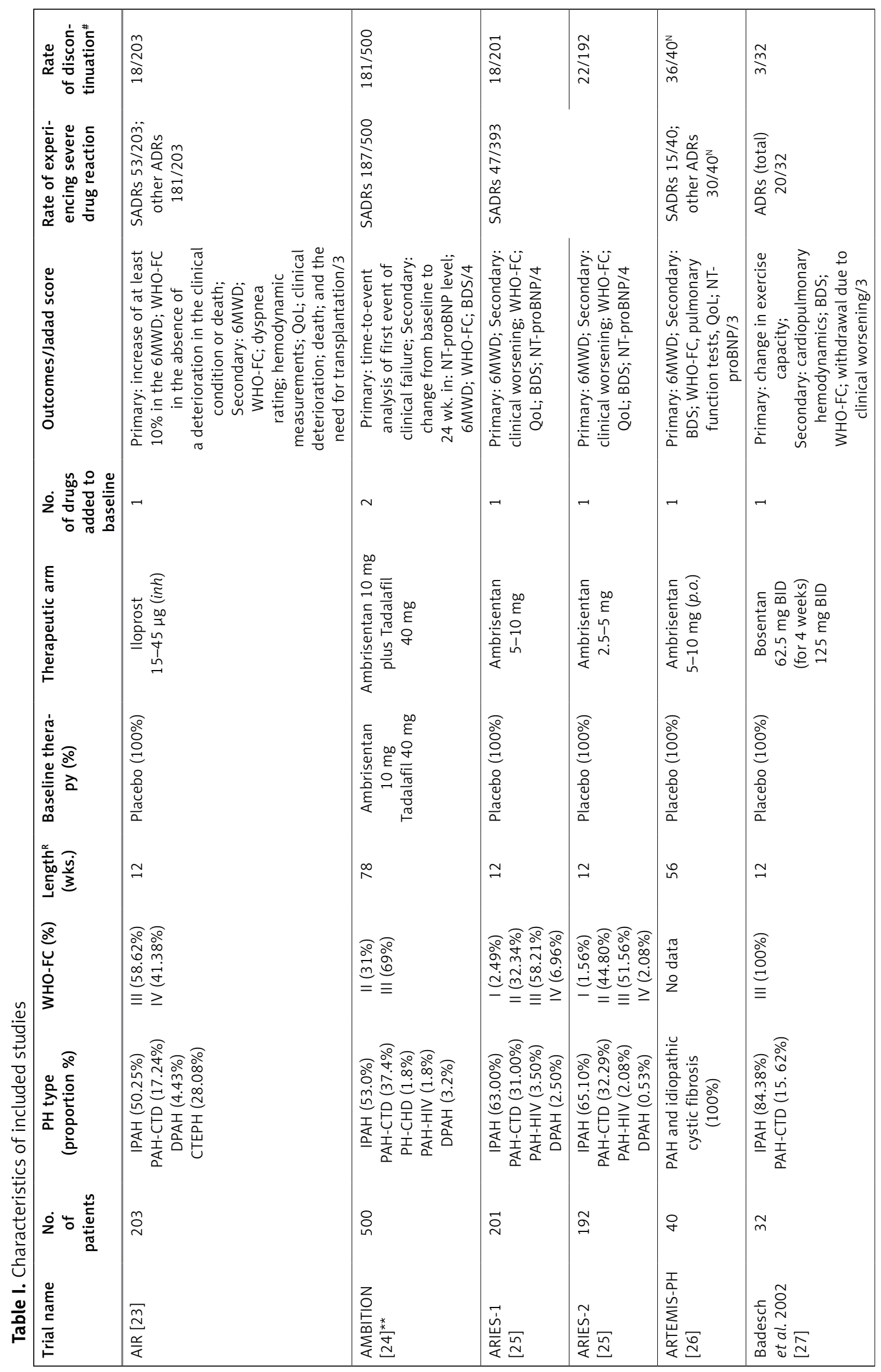




\begin{tabular}{|c|c|c|c|c|c|c|}
\hline 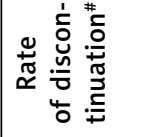 & 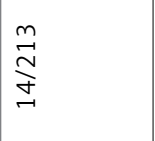 & 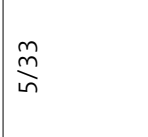 & 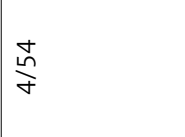 & 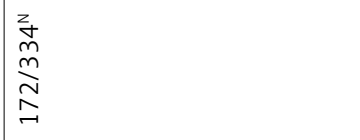 & 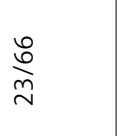 & 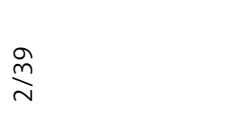 \\
\hline 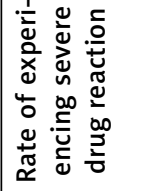 & 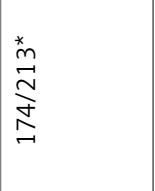 & 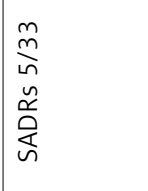 & 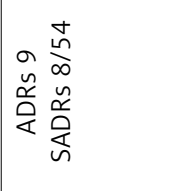 & 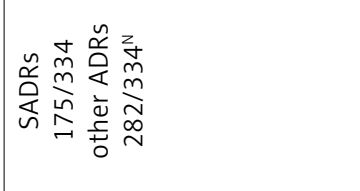 & 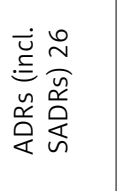 & 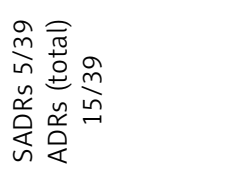 \\
\hline 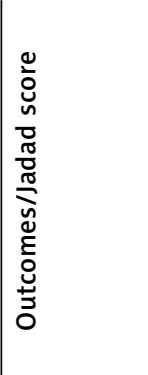 & 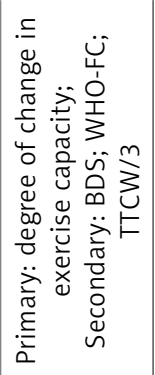 & 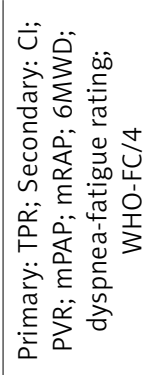 & 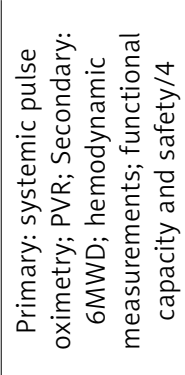 & 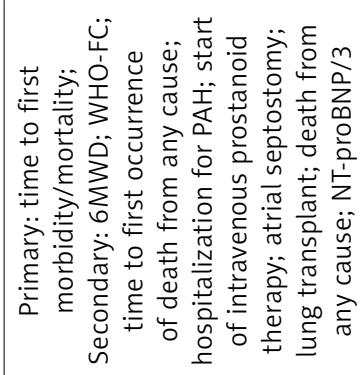 & 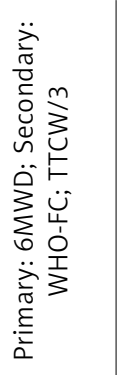 & 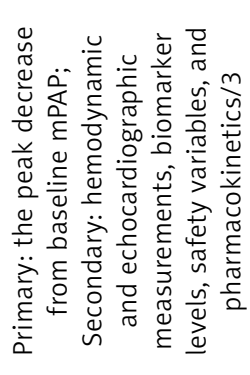 \\
\hline 总 & $\rightarrow$ & $\sim$ & $r$ & $\rightarrow$ & - & $\rightarrow$ \\
\hline 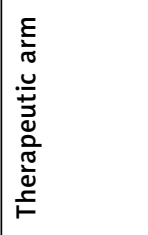 & 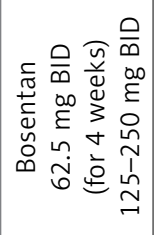 & 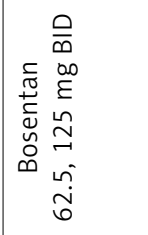 & 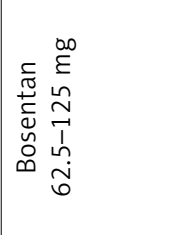 & 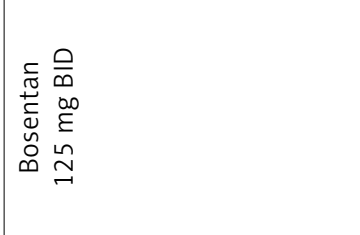 & 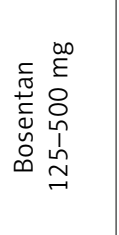 & 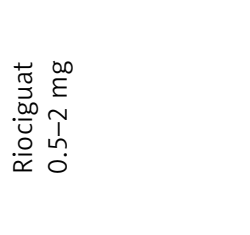 \\
\hline 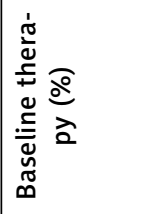 & 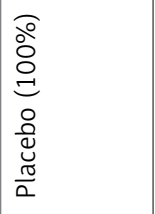 & 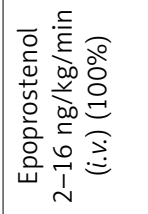 & 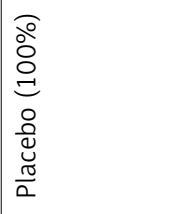 & 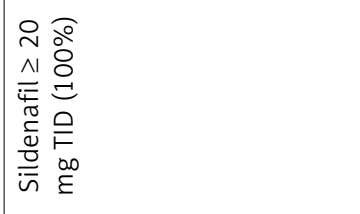 & 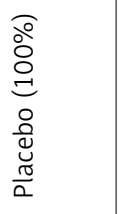 & 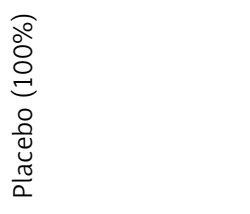 \\
\hline 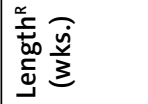 & $\stackrel{1}{1}$ & $\stackrel{0}{-1}$ & $\stackrel{2}{\longrightarrow}$ & $\stackrel{2}{2}$ & $\stackrel{0}{\sim}$ & $\stackrel{1}{\circ}$ \\
\hline 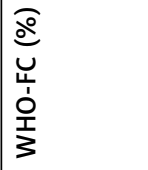 & 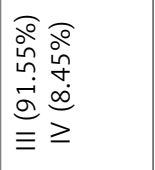 & 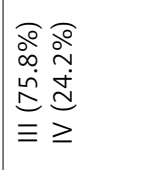 & $\begin{array}{l}\widehat{\circ} \\
\stackrel{\circ}{\stackrel{\circ}{\Xi}} \\
\equiv\end{array}$ & 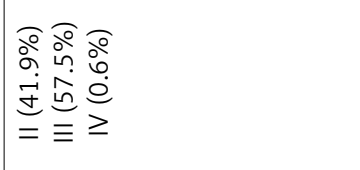 & 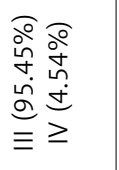 & $\begin{array}{l}\frac{\pi}{0} \\
\frac{\pi}{0} \\
0 \\
2\end{array}$ \\
\hline 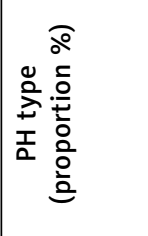 & 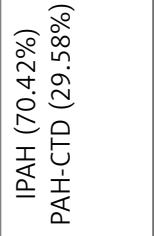 & 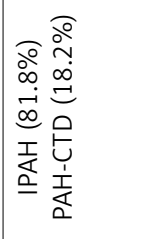 & $\begin{array}{l}\text { o̊ } \\
\text { Oे } \\
\stackrel{0}{0} \\
\text { 옳 } \\
\text { İ } \\
\text { I }\end{array}$ & 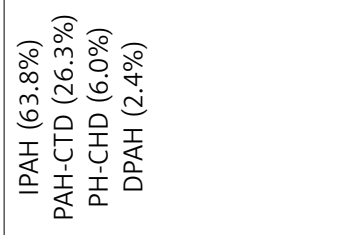 & 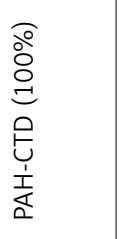 & 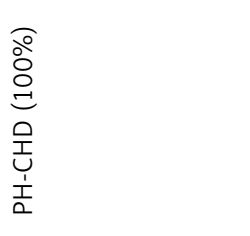 \\
\hline 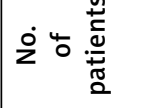 & $\stackrel{m}{\sim}$ & $\stackrel{m}{m}$ & in & $\underset{m}{\stackrel{N}{m}}$ & : & के \\
\hline 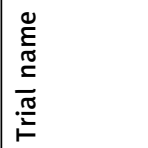 & 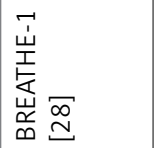 & 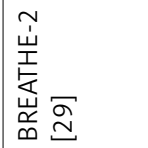 & 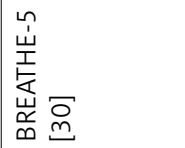 & 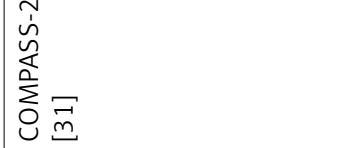 & 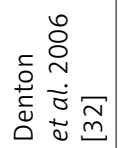 & $\stackrel{\text { 崖 }}{\bar{m}}$ \\
\hline
\end{tabular}




\begin{tabular}{|c|c|c|c|c|c|c|}
\hline 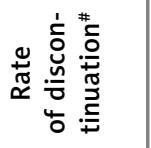 & $\stackrel{\substack{\infty \\
\stackrel{\infty}{\sim}}}{\stackrel{1}{\sim}}$ & $\begin{array}{l}\text { बे } \\
\text { ô. } \\
\text { ô }\end{array}$ & 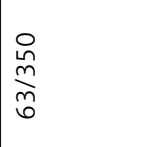 & $\begin{array}{l}\stackrel{\circ}{m} \\
\stackrel{m}{o} \\
\stackrel{g}{q}\end{array}$ & 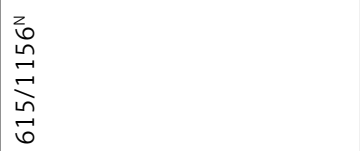 & $\underset{I}{\vec{I}}$ \\
\hline 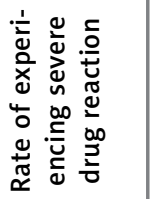 & 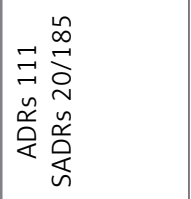 & 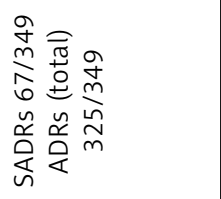 & 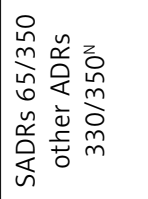 & 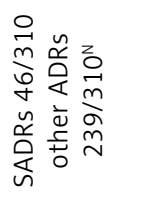 & 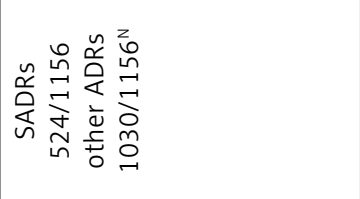 & 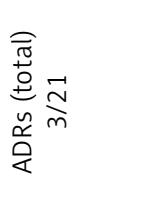 \\
\hline 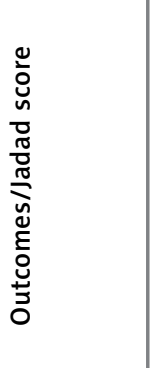 & 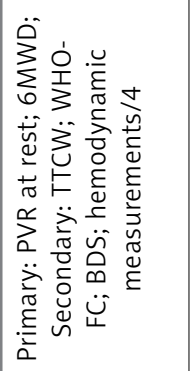 & 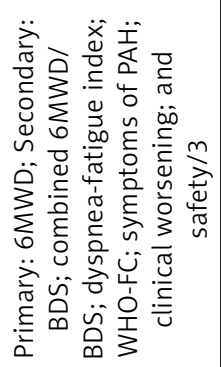 & 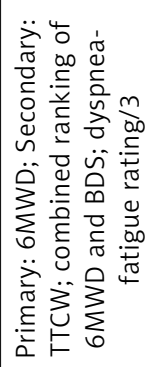 & 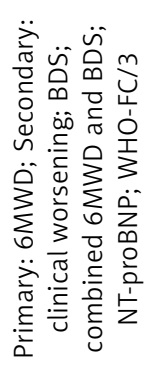 & 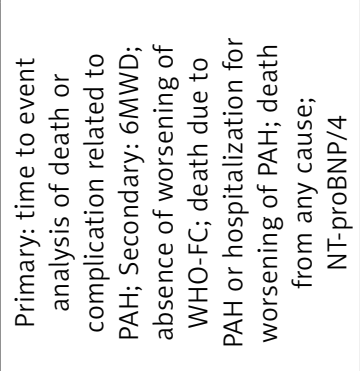 & 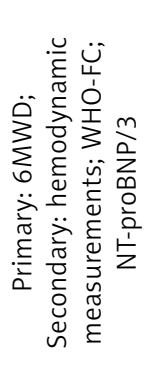 \\
\hline 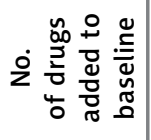 & - & $\rightarrow$ & - & $\rightarrow$ & - & $\rightarrow$ \\
\hline 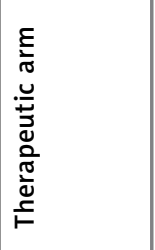 & 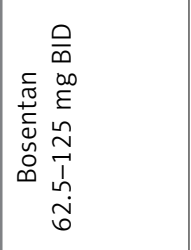 & 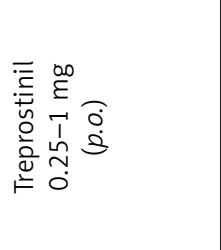 & 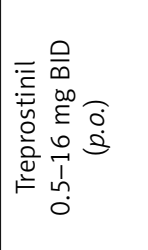 & 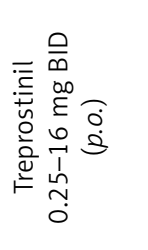 & 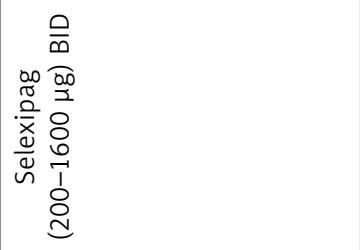 & 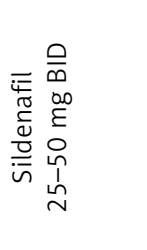 \\
\hline 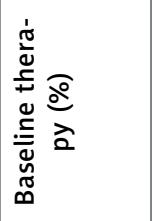 & 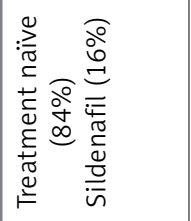 & 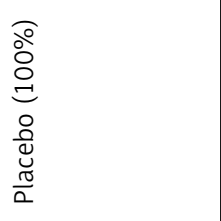 & 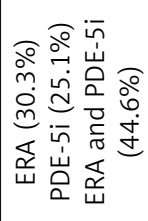 & 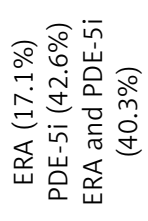 & 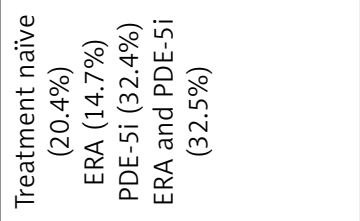 & 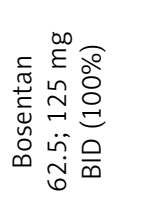 \\
\hline 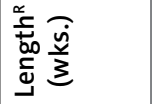 & $\stackrel{\sim}{\sim}$ & $\approx$ & $\stackrel{-}{-1}$ & $\stackrel{\circ}{\sim}$ & $\vec{\wedge}$ & $\stackrel{\dot{N}}{ }$ \\
\hline 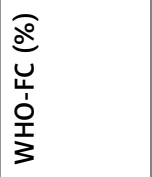 & $\begin{array}{l}\text { Oें } \\
\stackrel{0}{\stackrel{⿹}{=}}\end{array}$ & 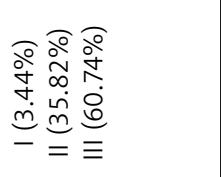 & 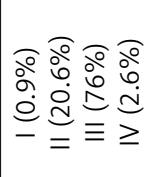 & 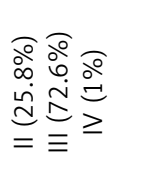 & 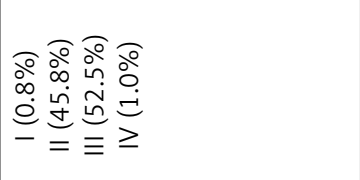 & 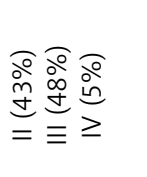 \\
\hline 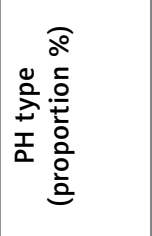 & 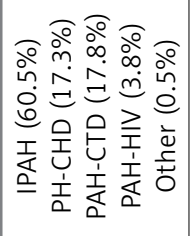 & 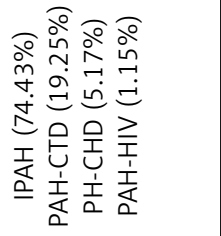 & 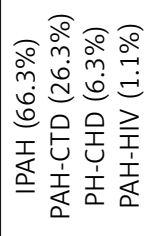 & 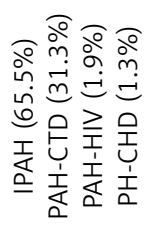 & 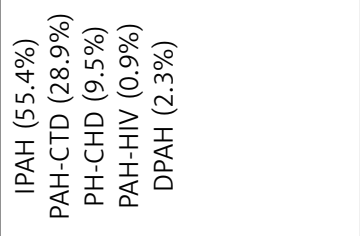 & 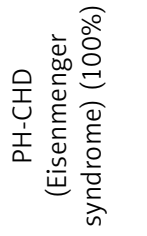 \\
\hline 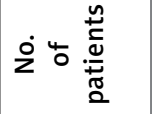 & $\stackrel{\leftrightarrow}{\sim}$ & $\underset{m}{\stackrel{g}{m}}$ & 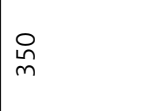 & $\stackrel{\vec{m}}{m}$ & 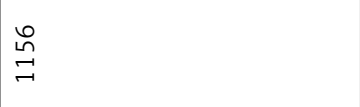 & $\vec{\sim}$ \\
\hline 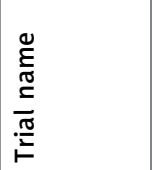 & 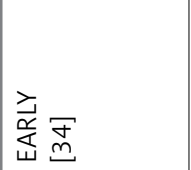 & 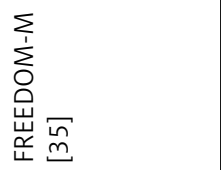 & 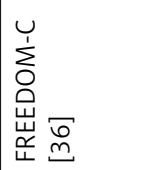 & 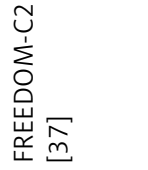 & 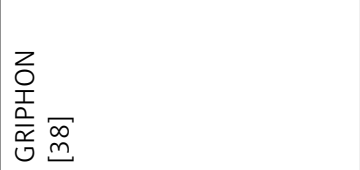 & 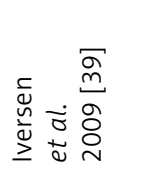 \\
\hline
\end{tabular}




\begin{tabular}{|c|c|c|c|c|c|c|}
\hline 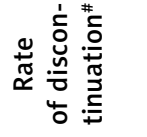 & 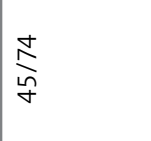 & $\underset{\substack{0 \\
\stackrel{n}{\sim}}}{\stackrel{\sim}{\sim}}$ & $\stackrel{\infty}{\longrightarrow}_{=}^{\infty}$ & 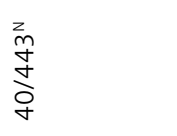 & $\underset{\substack{n\\
}}{\stackrel{2}{1}}$ & 望 \\
\hline 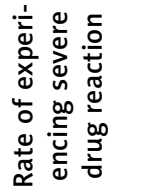 & 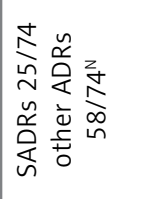 & 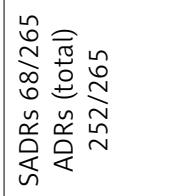 & 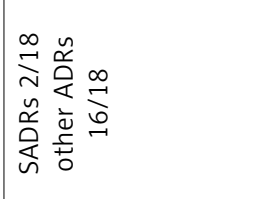 & 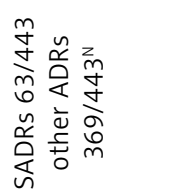 & 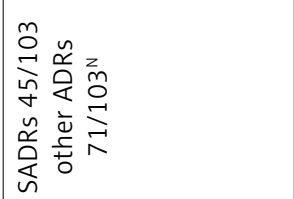 & 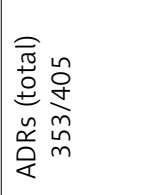 \\
\hline 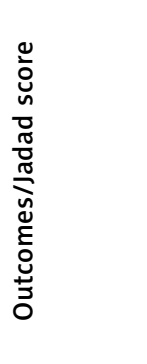 & 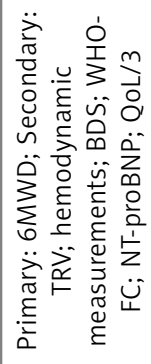 & 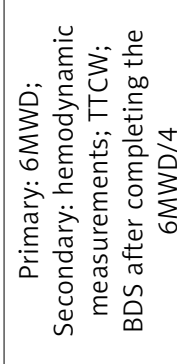 & 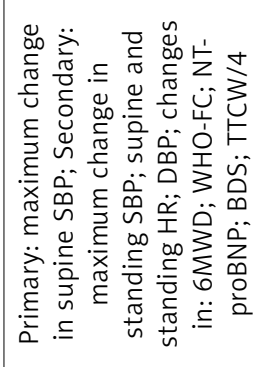 & 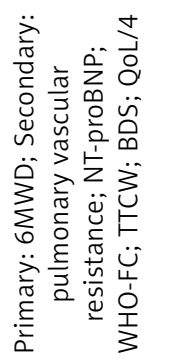 & 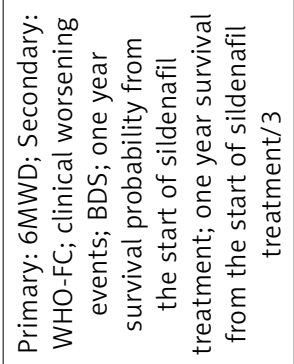 & 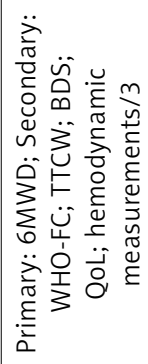 \\
\hline 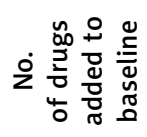 & $\rightarrow$ & - & - & - & $\rightarrow$ & $\neg$ \\
\hline 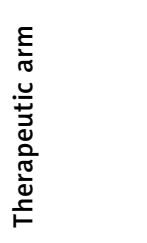 & 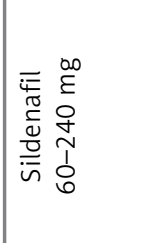 & 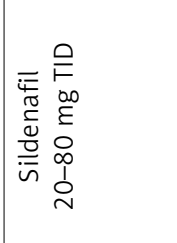 & 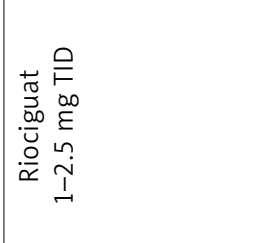 & 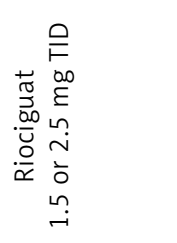 & 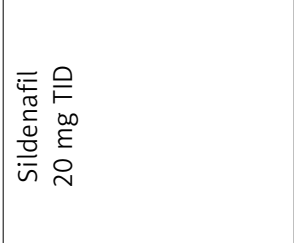 & 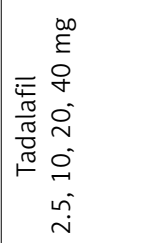 \\
\hline 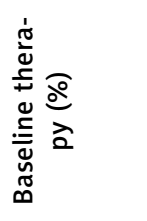 & 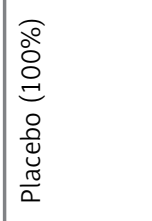 & 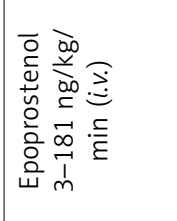 & 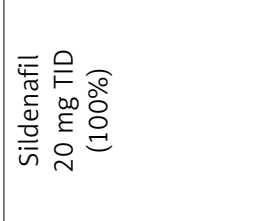 & 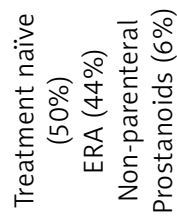 & 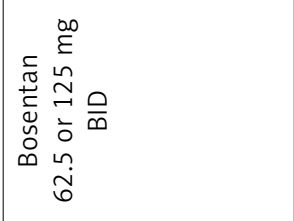 & 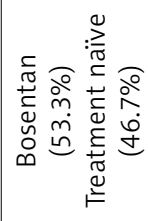 \\
\hline 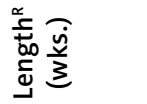 & $\stackrel{0}{\sim}$ & $\stackrel{0}{0}$ & $\underset{\sim}{\sim}$ & $\simeq$ & $\underset{\sim}{\sim}$ & $\stackrel{0}{\sim}$ \\
\hline 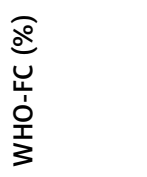 & $\begin{array}{l}\frac{\pi}{10} \\
\frac{\pi}{0} \\
2 \\
2\end{array}$ & 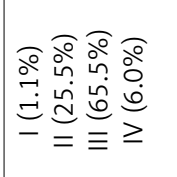 & 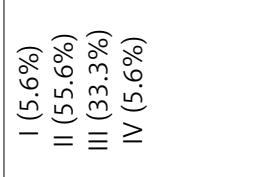 & 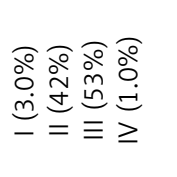 & 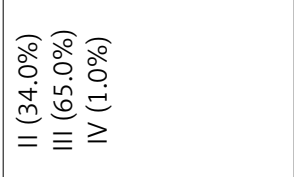 & 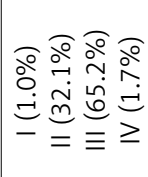 \\
\hline 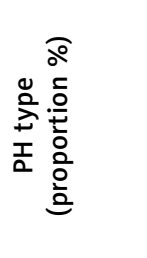 & 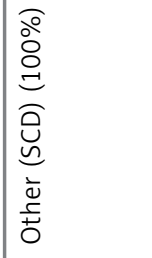 & 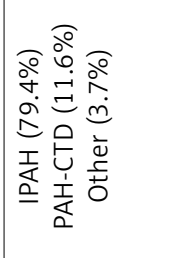 & 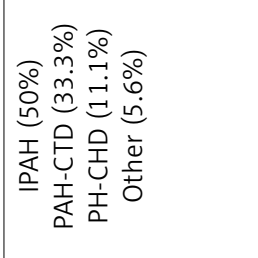 & 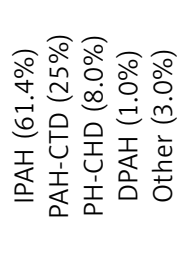 & $\begin{array}{l}\frac{\pi}{0} \\
\frac{\pi}{0} \\
0 \\
0 \\
0 \\
1 \\
0 \\
\frac{1}{1} \\
\frac{1}{\alpha}\end{array}$ & 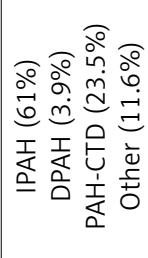 \\
\hline 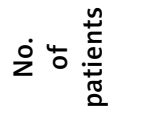 & I & $\stackrel{\sim}{\sim} \underset{\sim}{\sim}$ & $\stackrel{\infty}{\rightarrow}$ & $\stackrel{m}{f}$ & $\stackrel{m}{\oplus}$ & 朵 \\
\hline 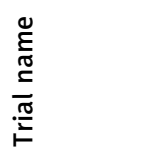 & 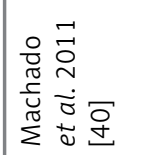 & 岂 & 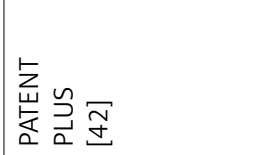 & 忿 & 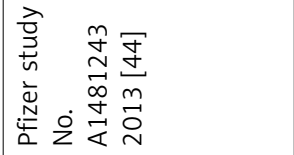 & 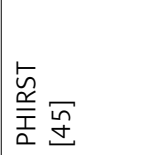 \\
\hline
\end{tabular}




\begin{tabular}{|c|c|c|c|c|c|c|c|}
\hline 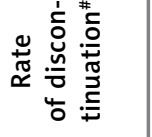 & $\underset{\sigma}{\stackrel{\Xi}{N}}$ & $\stackrel{\underset{N}{N}}{\text { N }}$ & 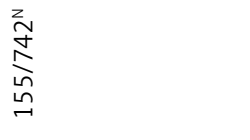 & $\frac{a}{\frac{a}{d}}$ & $\frac{m}{f}$ & $\frac{\hat{\sigma}}{a}$ & $\approx$ \\
\hline 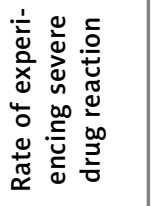 & 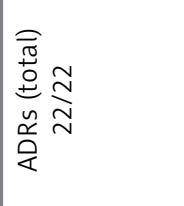 & 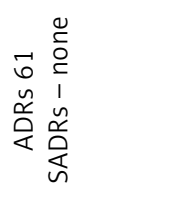 & 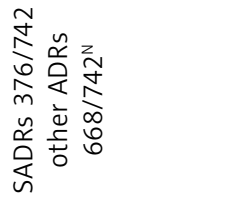 & 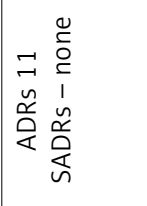 & 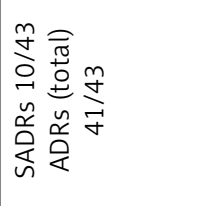 & 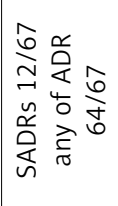 & 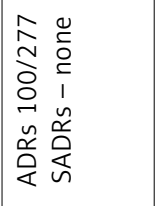 \\
\hline 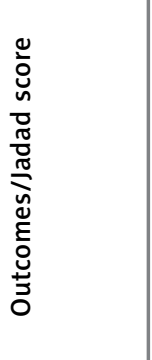 & 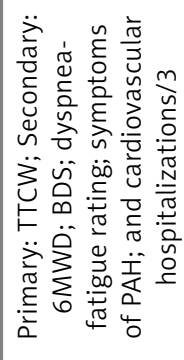 & 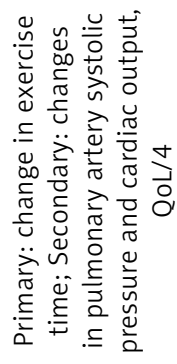 & 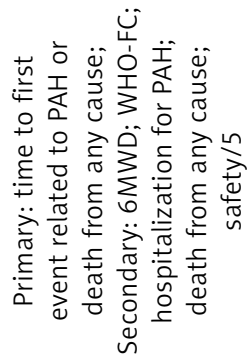 & 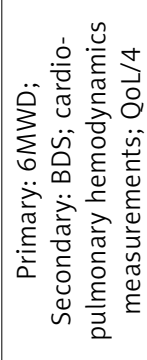 & 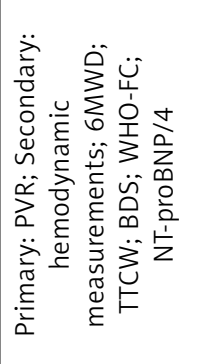 & 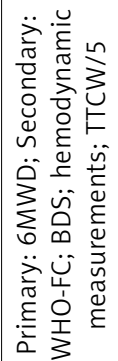 & 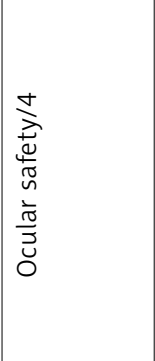 \\
\hline 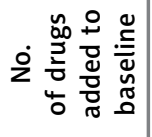 & - & - & $r$ & $\rightarrow$ & $\rightarrow$ & - & - \\
\hline 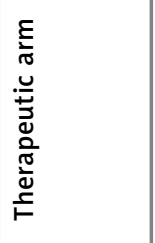 & 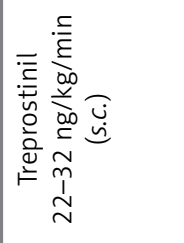 & 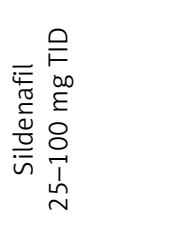 & 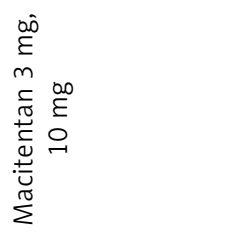 & 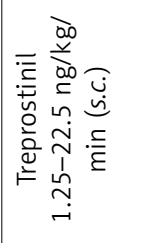 & 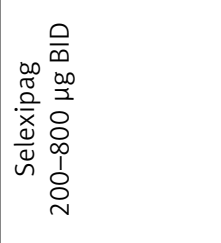 & 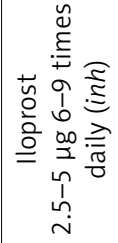 & 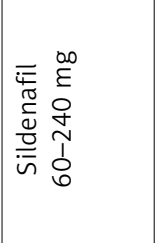 \\
\hline 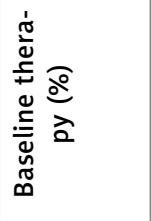 & 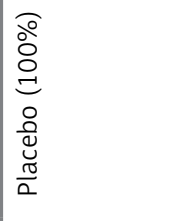 & 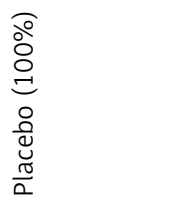 & 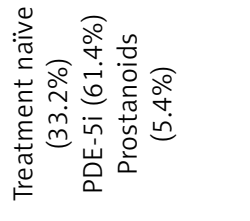 & 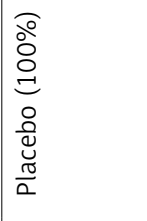 & 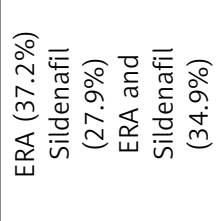 & 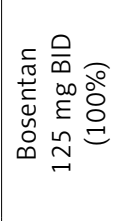 & 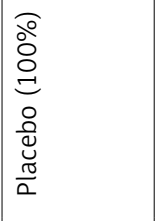 \\
\hline 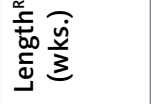 & $\infty$ & $\approx$ & 弚 & $\approx$ & 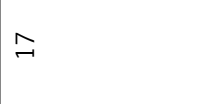 & $\approx$ & $\approx$ \\
\hline 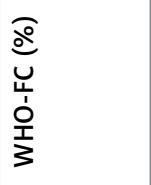 & 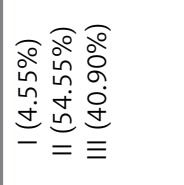 & 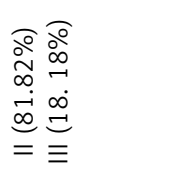 & 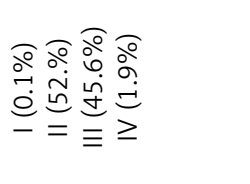 & 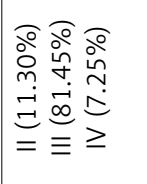 & 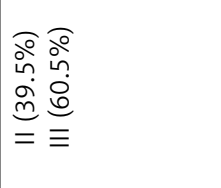 & 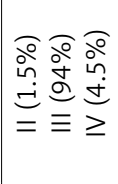 & 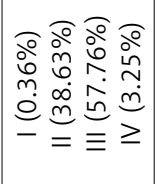 \\
\hline 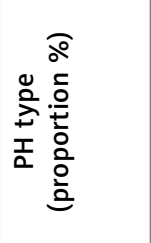 & 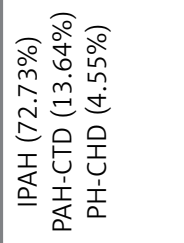 & 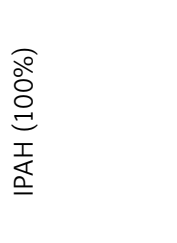 & 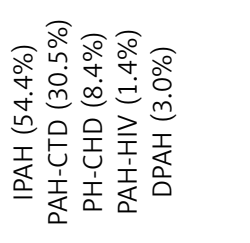 & 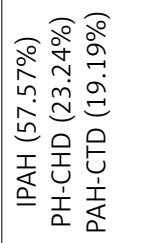 & 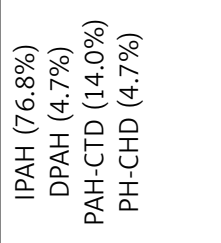 & 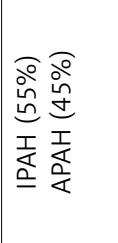 & 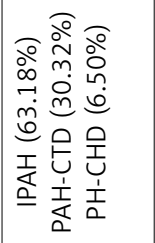 \\
\hline 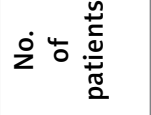 & $\tilde{N}$ & $\tilde{N}$ & $\stackrel{\mathcal{Z}}{\mathcal{Z}}$ & $\begin{array}{l}a \\
\text { oे }\end{array}$ & $\stackrel{m}{\forall}$ & $\widehat{6}$ & $\stackrel{\aleph}{\lesssim}$ \\
\hline 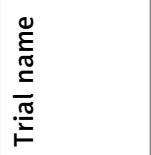 & 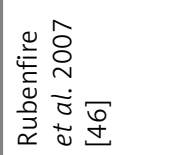 & 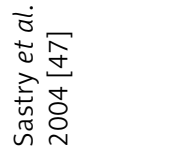 & 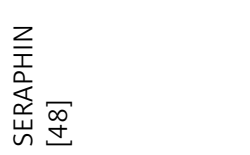 & 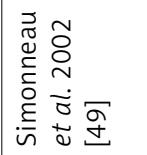 & 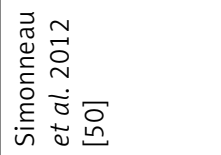 & 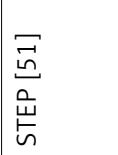 & 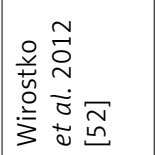 \\
\hline
\end{tabular}




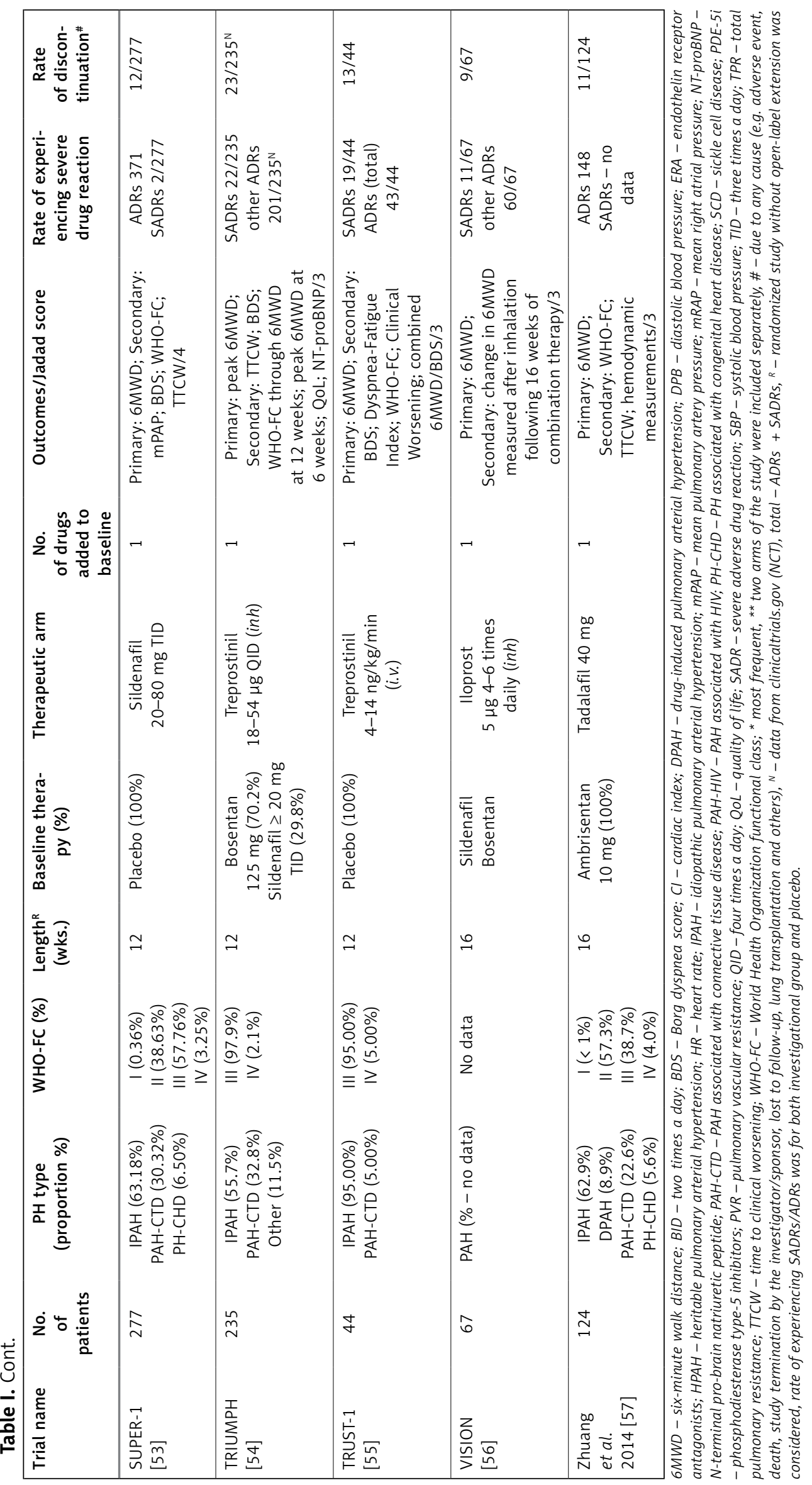


Among them we assessed the increased risk of adverse effects resulting from addition to the background (placebo or aimed therapy) of the following targeted strategies: (i) endothelin receptor antagonist (ERA): bosentan, ambrisentan or macitentan ( $n=3590$ patients); (ii) phosphodiesterase type 5 inhibitor (PDE-5i): sildenafil or tadalafil ( $n$ = 3675); (iii) soluble guanylate cyclase stimulator (GCs): riociguat ( $n=354)$; (iiii) prostacyclins $\left(\mathrm{PGI}_{2}\right)$ : treprostinil (p.o., i.v., s.c.), or iloprost (inh) $(n=1515)$ or selective prostacyclin receptor agonist (selexipag) $(n=607)$ within a 2 to 26 month-period. The primary and secondary outcomes included: 6-minute walk distance (6MWD); time to clinical worsening (TTCW); hemodynamic parameters: systolic (diastolic) blood pressure; heart rate (HR) and pulmonary vascular resistance (PVR) or $\mathrm{N}$-terminal pro-brain natriuretic peptide (NT-proBNP) (Table I).

\section{Discontinuations and severe drug reactions}

Discontinuations because of adverse events were reported in 30 per 36 trials included in the meta-analysis. Overall, no significant impact on the increased risk of discontinuations due to ADR was identified $(R R=0.99,95 \% \mathrm{Cl}: 0.74-1.32$, $\left.p=0.9443, I^{2}=50.88 \%\right)$. Further subgroup analysis revealed some increased risk only when the IP agonist selexipag was added to the baseline therapy $\left(\mathrm{RR}=2.01,95 \% \mathrm{Cl}: 1.41-2.86, p<0.0001, \mathrm{Q}_{\text {inter-group, }}\right.$ 17.53, $\mathrm{d} f=4, p=0.0015)$, not the other agents (Figure 2A). Route of administration or therapeutic regimen was also not found to have any significant impact on the altered risk. Patients receiving the combined regimen demonstrated a greater tendency of risk cessation $\left(\mathrm{Q}_{\text {inter-group }}, p=0.0779\right)$. Such risk of discontinuation was increased particularly when bosentan (ERA) $(\mathrm{RR}=1.64,95 \% \mathrm{Cl}: 1.11-$ 2.43, $p<0.05)$, treprostinil $\left(\mathrm{PGI}_{2}\right)(\mathrm{RR}=3.27,95 \%$ $\mathrm{Cl}: 1.28-8.34, p<0.05)$ or selexipag (IP agonist) $(\mathrm{RR}=2.01,95 \% \mathrm{Cl}: 1.41-2.86, p<0.05)$ was added to the baseline $\left(\mathrm{Q}_{\text {inter-group }}, p<0.05\right)$ (Figure $\left.2 \mathrm{~B}\right)$.

Severe drug reactions were reported in 25 out of 36 trials. No significant impact on the increased risk of severe ADR was found (RR $=0.97,95 \% \mathrm{Cl}$ : 0.84$\left.1.13, p=0.7210, P^{2}=25.84 \%\right)$. We did not observe any discrepancies according to particular agent or therapeutic group (Figure 2C). No other factors such as route of administration or therapeutic regimen (i.e. one, two or three drugs added to baseline) were revealed to determine the effect size.

\section{Adverse drug reactions (other than severe ADRs)}

When considering all significant ADRs as a whole, subjects who used monotherapy experienced increased risk of adverse reactions
$(\mathrm{RR}=1.44,95 \% \mathrm{Cl}: 1.27-1.64, p<0.0001 ; \mathrm{com}-$ parator: placebo/supportive therapy). Similarly, in patients receiving combined therapy the relative risk was significantly increased $(R R=1.42,95 \%$ $\mathrm{Cl}$ : 1.32-1.54, $p<0.0001$; active comparator $\mathrm{PH}$-specific agent). A significant tendency toward increased RR was observed for such ADRs as blood and lymphatic system disorders with the anemia subgroup, gastrointestinal disorders with diarrhea and nausea subgroups, respiratory and thoracic diseases, nervous system disorders with headache, vascular events, myalgia and pain in limb. The first four main categories of ADRs tended to occur more often in combination regimens as compared to monotherapy (Figure 2D).

The overall frequency for events classified into the remaining categories (e.g. blood and lymphatic system, the gastrointestinal, musculoskeletal or nervous systems, or respiratory or vascular disorders) was confirmed to be high (SmPC categories: common or very common). Figure 3 presents results for ADRs. A detailed description is provided in Supplementary Material (Results).

\section{Publication bias}

A visual inspection of funnel plots and Egger's test were used to evaluate publication bias (Figure 4).

\section{Sensitivity analysis}

In relation to the majority of reported ADRs, the observed effect was robust in sensitivity analysis and the statistical significance was not influenced by any single study included in the meta-analysis. Table II summarizes the sensitivity analysis with the most frequent subcategories of ADRs that were reported in studies included in the meta-analysis.

\section{Discussion}

At the time of writing, this was the first metaanalysis of 7977 participants intended to compare the safety profile of particular $\mathrm{PH}$-specific therapies according to individual risk of adverse drug reaction based on a review of randomized trials using both placebo-controlled and active comparators.

Our main finding is that individual $\mathrm{PH}$-targeted therapies do not significantly enhance the risk of either discontinuations due to ADRs or severe adverse drug reactions. The risk of cessation was comparable between patients receiving $\mathrm{PH}$-specific agents in monotherapy or combination, with some tendency toward the combined regimen. In the subgroup of patients receiving combined therapy, the relative risk of discontinuation due to adverse events was significantly increased when tre- 
A

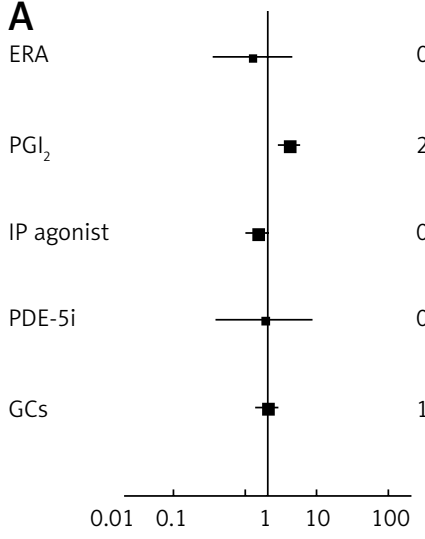

$\underset{\text { ERA }}{C}$

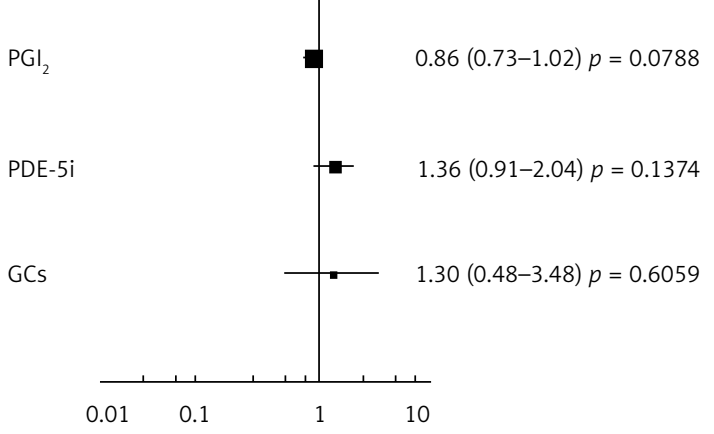

D

Blood and lymphatic diseases

Anemia

Gastrointestinal diseases

Diarrhea

Nausea

Musculoskeletal and connective tissue diseases

Muscle pain

Jaw pain

Pain in limb

Nervous system diseases

Headache

Respiratory and thoracic diseases

Epistaxis

Vascular diseases

Flushing

Eye disorders

Peripheral edema

$01(0.52-1.96) p=0.9698$
$2.01(1.41-2.86) p=0.0001$

$0.73(0.51-1.03) p=0.0762$

$0.93(0.20-4.37) p=0.9271$

$1.00(0.72-1.39) p=0.9964$

B

Ambrisentan

Macitentan

Sildenafil

Tadalafil

Riociguat

lloprost

Treprostinil

Selexipag

$0.01 \quad 0.1$
$0.71(0.44-1.15) p=0.1631$

$1.64(1.11-2.43) p=0.0127$

$0.98(0.65-1.47) p=0.9205$

$0.70(0.35-1.39) p=0.3089$

$0.67(0.43-1.04) p=0.0751$

$1.60(0.27-9.45) p=0.6050$

$0.28(0.03-3.10) p=0.3013$

$3.27(1.28-8.34) p=0.0133$

$2.01(1.41-2.86) p=0.0001$
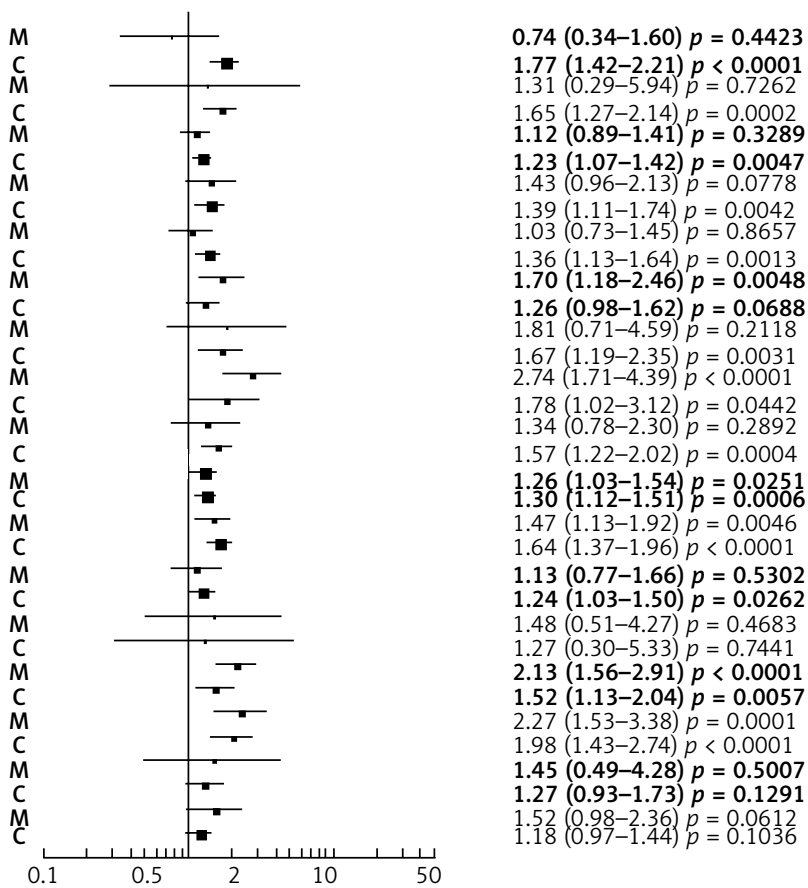

Figure 2. Effect of pulmonary hypertension ( $\mathrm{PH}$ )-specific agents on the relative risk (RR, $95 \% \mathrm{Cl}$ ): $\mathrm{A}$ - Discontinuations due to adverse drug reactions (ADRs) $-\mathrm{RR}=0.99,95 \% \mathrm{Cl}: 0.74-1.32, p=0.9443, I^{2}=50.88 \%$; $\mathrm{B}$ - Discontinuations for combined regimens (subgroup analysis). The relative risk of discontinuation was increased particularly when bosentan, treprostinil or selexipag was added to the monotherapy; C - Severe ADRs - RR = 0.97, 95\% Cl: 0.84-1.13, $p=0.7210$; D - Categories of ADRs* (bold font) with selected subcategories. For combination regimens, a significant tendency toward increased RR was observed for such ADRs as blood and lymphatic system disorders with anemia subgroup, gastrointestinal disorders with diarrhea and nausea subgroups, myalgia and pain in limb, respiratory, vascular or nervous system disorders with headache subgroup. M- monotherapy (comparator: placebo/supportive therapy), C - combined therapy (active comparator: monotherapy). *only ADRs with significant result for relative risk were examined 
A

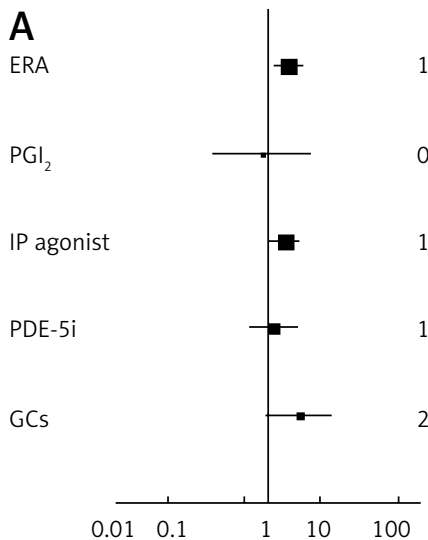

B

ERA

$\mathrm{PGI}_{2}$

IP agonist

PDE-5i

GCs

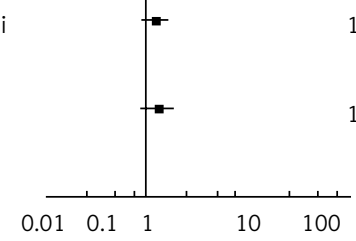

B2

ERA

$\mathrm{PGI}_{2}$

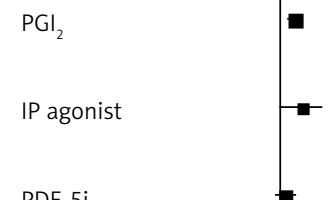

PDE-5i

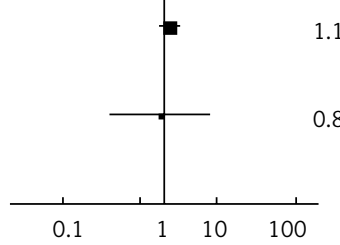

C1

ERA

$\mathrm{PGI}_{2}$

IP agonist

PDE-5i

GCS

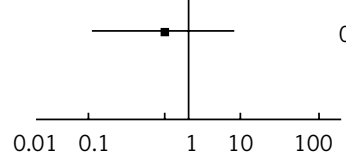

GCs
$1.84(1.21-2.79) p=0.0043$

$0.84(0.19-3.69) p=0.8227$

$1.65(1.08-2.52) p=0.0213$

$1.19(0.59-2.41) p=0.6325$

$2.55(0.92-7.05) p=0.0722$
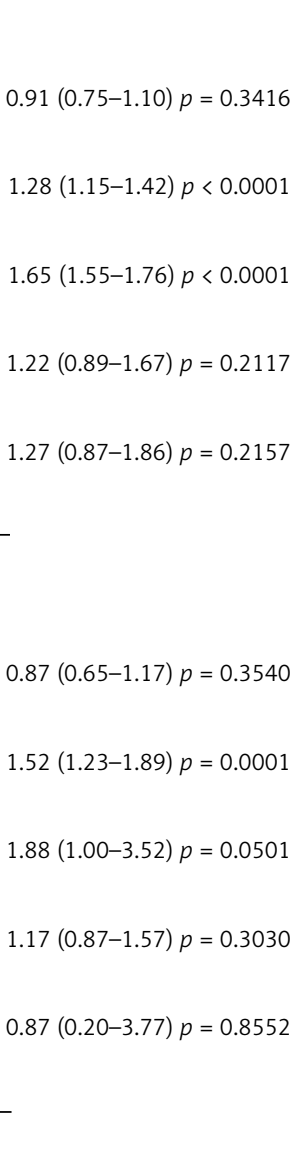

\section{C}

ERA

$\mathrm{PGI}_{2}$

IP agonist

B1

ERA

$\mathrm{PGI}_{2}$

IP agonist

PDE-5i

GCS
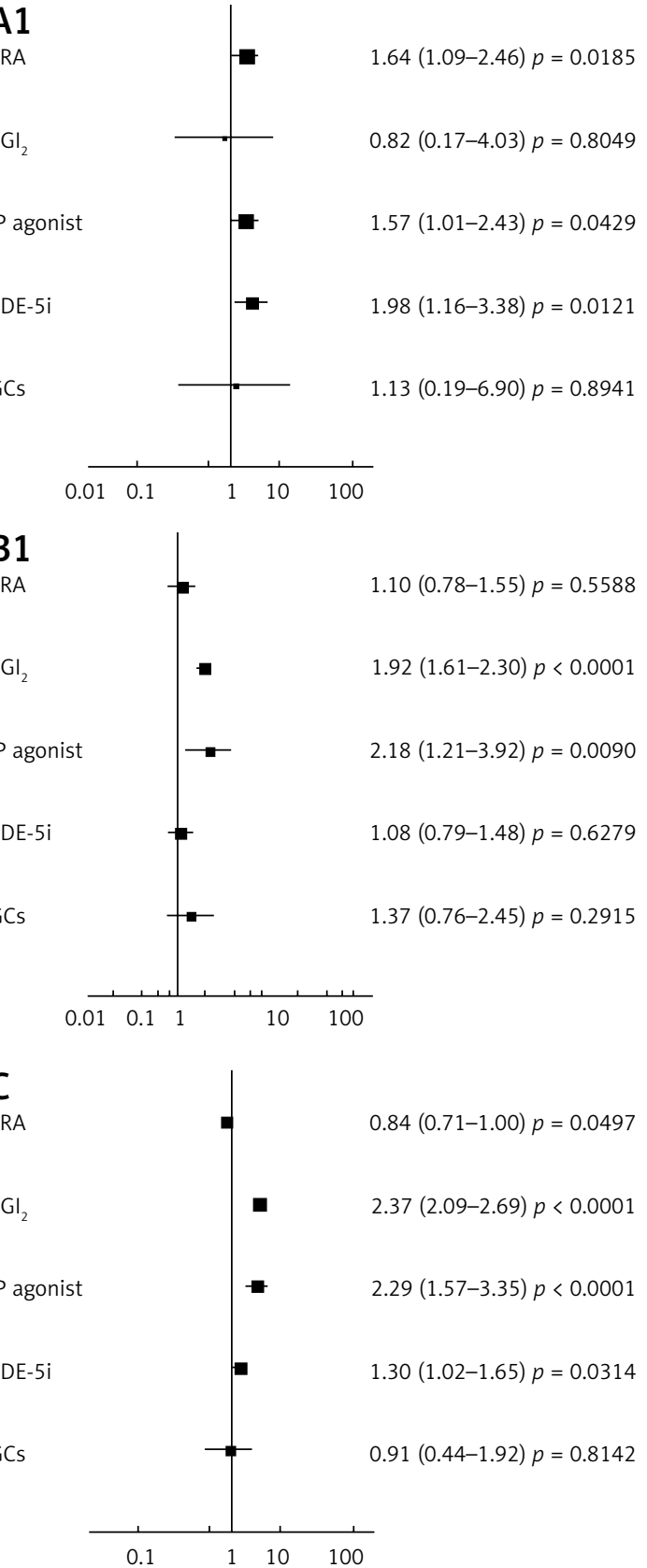

$\mathrm{C} 2$

$0.83(0.50-1.40) p=0.4929 \quad$ ERA

$2.35(1.36-4.05) p=0.0022 \quad P G I_{2}$

$2.74(1.88-4.00) p<0.0001 \quad$ IP agonist

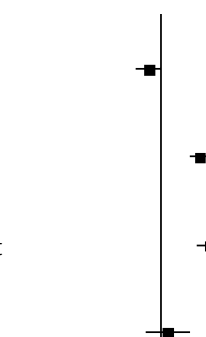

$0.69(0.47-1.02) p=0.0619$

$3.08(2.35-4.03) p<0.0001$

$4.13(2.93-5.81) p<0.0001$

$1.20(0.60-2.42) p=0.6087$

$0.47(0.05-4.06) p=0.4898 \quad$ GCs

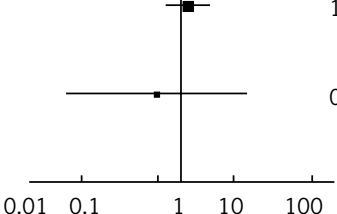

Figure 3. Effect of pulmonary hypertension ( $\mathrm{PH})$-specific agents on the relative risk $(\mathrm{RR}, 95 \% \mathrm{Cl})$ of selected adverse drug reactions (ADRs). The overall frequency of ADRs in the investigational groups was as follows: $\mathbf{A}-$ blood and lymphatic disorders (monotherapy: 8.53\% (baseline)/4.22\% (comparator) and combination: 4.74\% (baseline)/9.74\% (comparator), A1 - anemia (1.55\%/2.20\% and 3.38\%/6.19\%), B - gastrointestinal disorders $(25.80 \% / 32.19 \%$ and 55.40\%/69.57\%), B1 - diarrhea (11.20\%/16.59\% and 15.51\%/20.67\%), B2 - nausea (14.51\%/17.30\% and 14.99\%/18.49\%), C - musculoskeletal and connective tissue disorders ( $11.44 \% / 26.89 \%$ and $34.61 \% / 43.54 \%)$, C1 - muscle pain (1.69\%/5.07\% and 4.12\%/7.30\%), C2 - jaw pain (3.69\%/12.18\% and 4.40\%/10.47\%) 

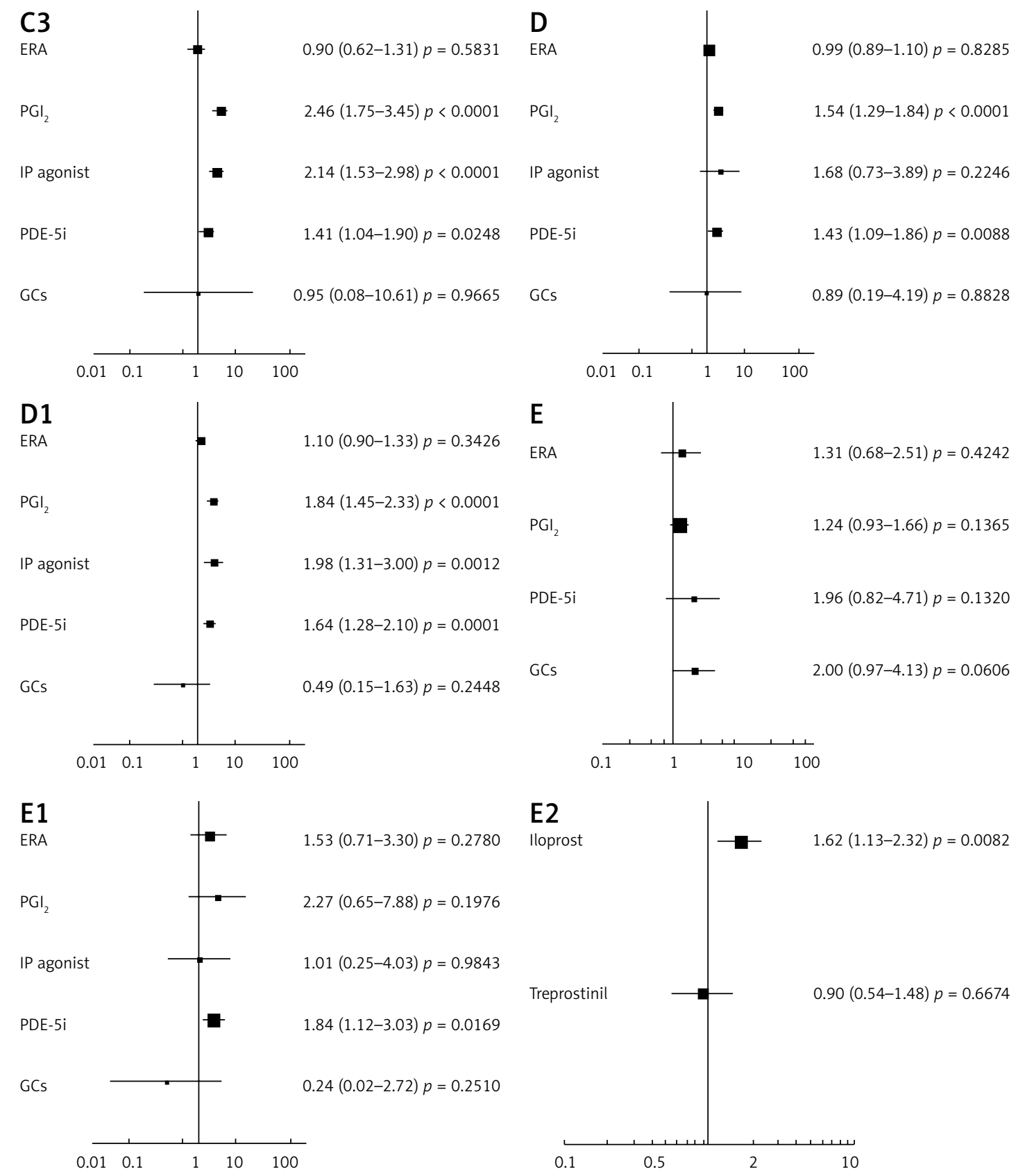

E2

Iloprost $\quad-\quad 1.62(1.13-2.32) p=0.0082$
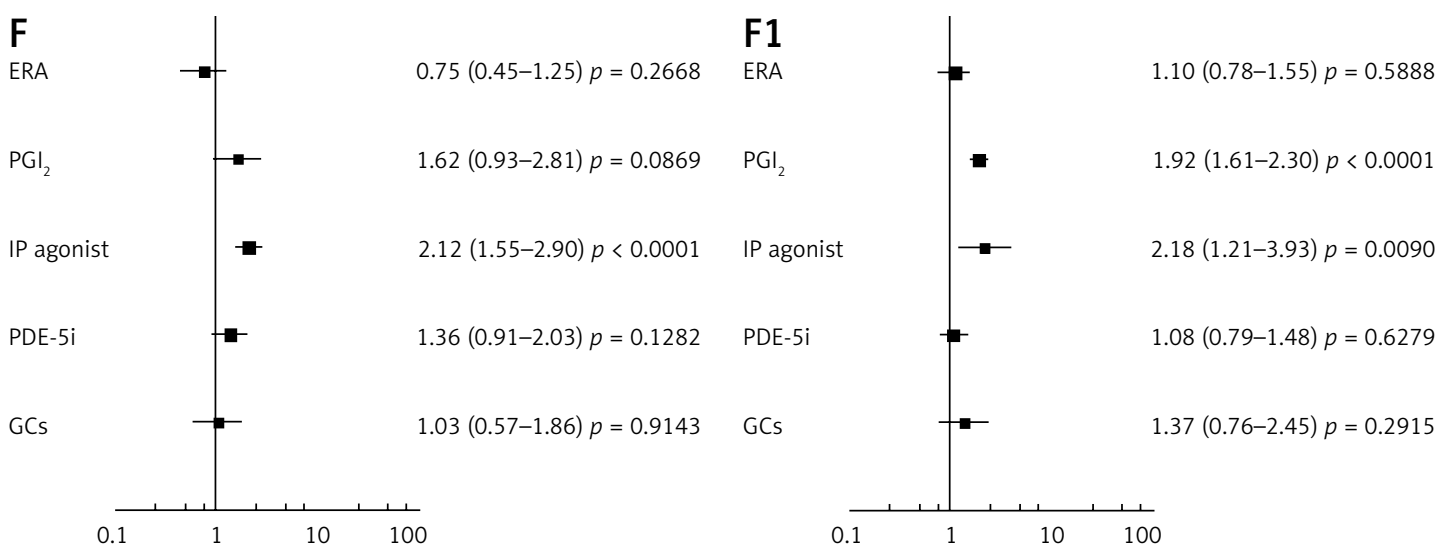

Figure 3. Cont. C3 - pain in limb (7.47\%/10.67\% and 7.31\%/11.09\%), D - nervous system disorders (27.70\%/37.39\% and $46.83 \% / 58.79 \%)$, D1 - headache (20.37\%/32.04\% and $22.34 \% / 32.95 \%)$, E - respiratory and thoracic system disorders (19.82\%/19.73\% and 56.26\%/70.40\%), E1 - epistaxis 1.27\%/4.02\% and 2.58\%/3.62\%), E2 - cough (subgroup analysis: $\mathrm{PGI} \mathrm{I}_{2}$ ), $\mathrm{F}$ - vascular disorders (5.79\%/11.22\% and 9.90\%/17.14\%), F1 - flushing (4.75\%/10.75\% and $6.02 \% / 12.09 \%)$ 


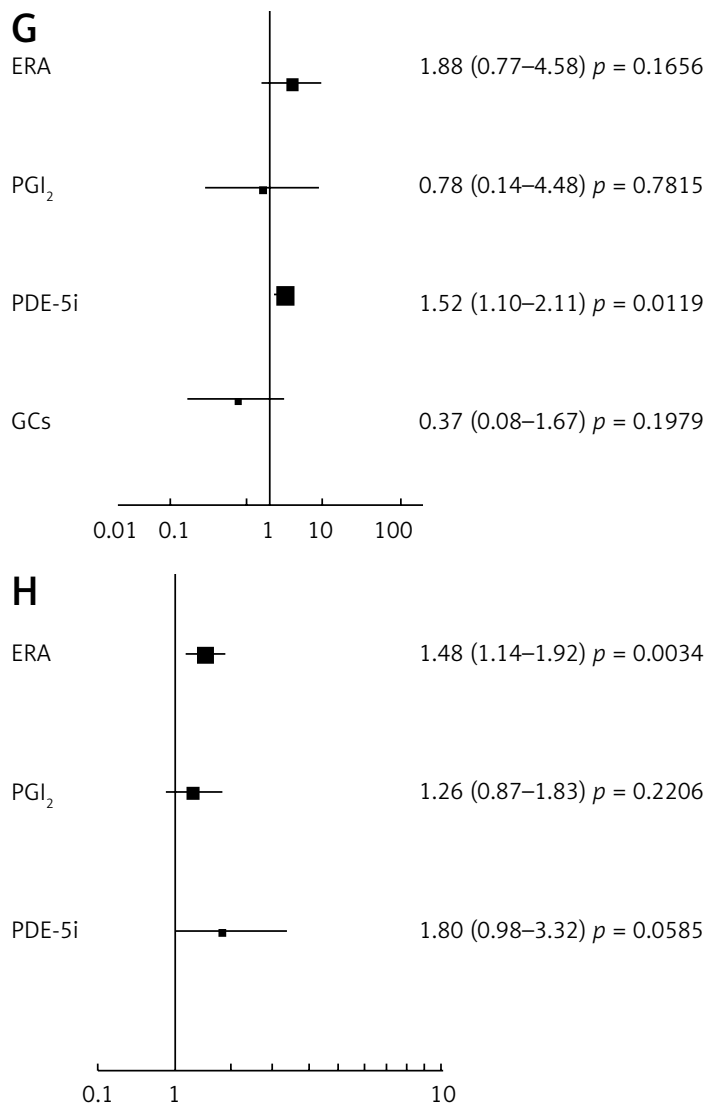

Figure 3. Cont. G - eye disorders (8.74\%/14.91\% and 0.84\%/2.05\%), G1 - subgroup analysis: PDE- 5 i, H - peripheral edema (7.26\%/12.58\% and $12.65 \% / 15.49 \%)$. Significantly increased risk was denoted for overall category (A) and for the anemia events subcategory (A1). The risk of any gastrointestinal disorders was slightly increased (B) with predominant contribution of prostacyclins $\left(\mathrm{PGI}_{2}\right)$ or IP agonist. The most prevalent were diarrhea (B1) and nausea (B2). The risk of musculoskeletal system-linked events was increased in a slight but statistically significant manner (C). Muscle pain, jaw pain and pain in limb were reported to significantly increase for selexipag and $\mathrm{PGI}_{2} \mathrm{~S}$ (C1-C3). In accordance with nervous system (D) or headache subcategory (D1) increased RR was observed when $\mathrm{PGI}_{2}$ or PDE-5i was added to baseline. Overall, the relative risk of respiratory and thoracic disorders was slightly, but significantly increased (E). Incidents of epistaxis (E1) mainly involving sildenafil were noted. Some increase of cough incidence in the subgroup of patients receiving iloprost was noted (E2). $\mathrm{PGI}_{2} \mathrm{~s}$ and selexipag were particularly superior to other agents for enhancing risk of vascular disorders (overall) (F) and flushing (F1). The risk of events classified as 'eye disorders' was slightly but significantly increased with the predominant contribution of PDE-5i: sildenafil (G-G1). An increased risk of peripheral edema was noted when ERAs were added to the baseline (H)

prostinil, selexipag or bosentan was added to the baseline. Likewise, in a previously published meta-analysis, it was found that treatment discontinuation was more likely to occur in patients receiving combined therapy, and this increased risk was particularly pronounced for non-parenteral prostaglandins and the selective prostacyclin receptor agonist [6]. As presented in Table II, the overall rate of discontinuations observed in some trials was high, about $30-50 \%$. These figures included events from any cause, with the most common ones being termination by sponsor or investigator (ARTEMIS-PH), withdrawal of consent (COMPASS-2), morbidity or mortality primary endpoint (GRIPHON), as well as loss of follow-up or lung transplantation, while the percentage of discontinuations due to adverse events was lower (5-15\%).

Both the present study and a previous one [14] suggest that the incidence of serious adverse events was similar between the monotherapy and combination regimen.

Of 26 main categories of ADRs defined by the MedRA Dictionary, 18 were not significantly affected by particular therapeutic agents as compared to baseline; these included benign neoplasms, disorders of the reproductive system and breast, infections or immune system disorders, or those linked to the endocrine system, kidney and urinary tract.

Conversely, the overall frequency for events classified into the remaining categories (e.g. blood and lymphatic system, the gastrointestinal, musculoskeletal or nervous systems, or the respiratory or vascular disorders) were confirmed to occur with high frequency $(>1 / 10$ or $>1 / 100)$. Particular $\mathrm{PH}$-specific therapies had the greatest effect on events associated with vasodilatory activity: flushing and headache as well as jaw pain, limb pain 
A

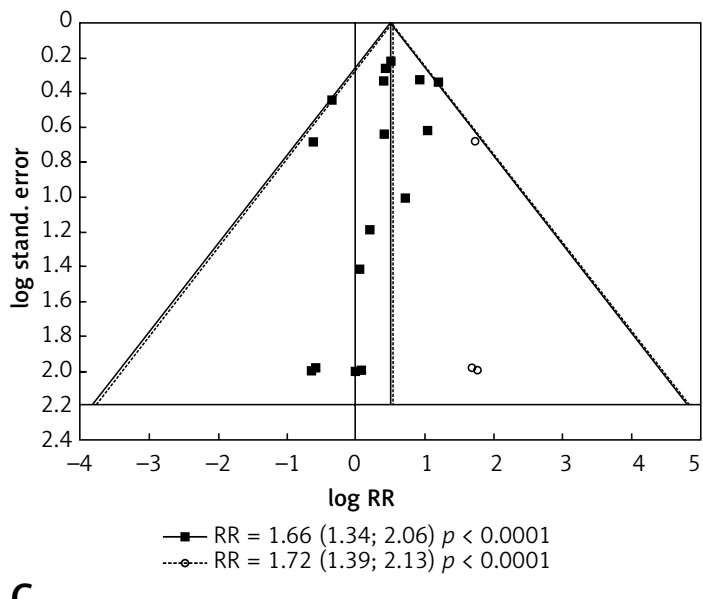

C

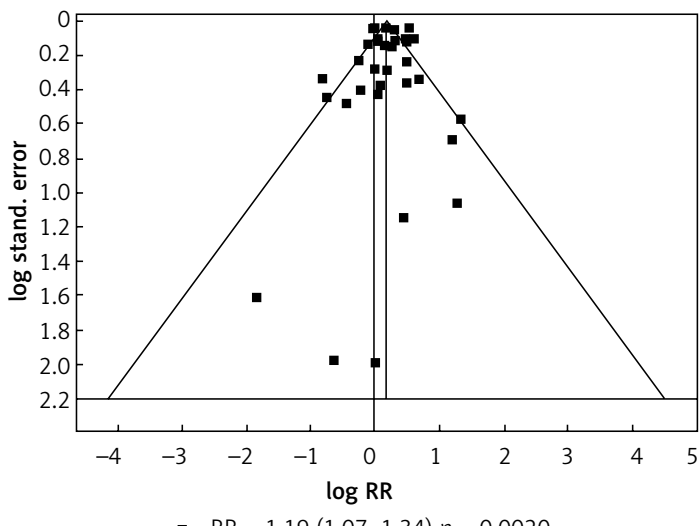

$R R=1.19(1.07 ; 1.34) p=0.0020$

E

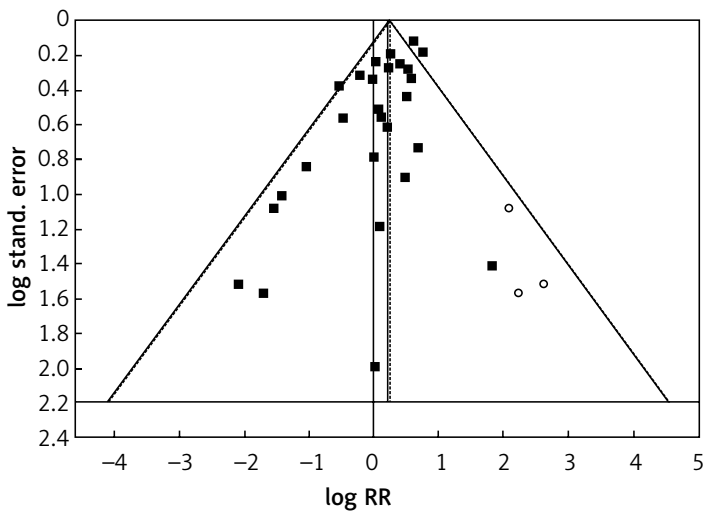

$\rightarrow-\mathrm{RR}=1.25(1.05 ; 1.49) p=0.0114$

$\ldots$..... $R R=1.27(1.06 ; 1.52) p=0.0081$
B

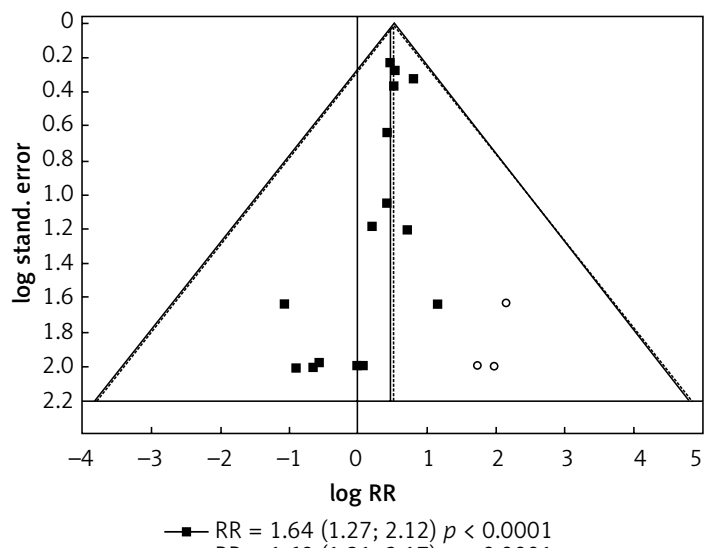

$\rightarrow \mathrm{RR}=1.64(1.27 ; 2.12) p<0.0001$

D

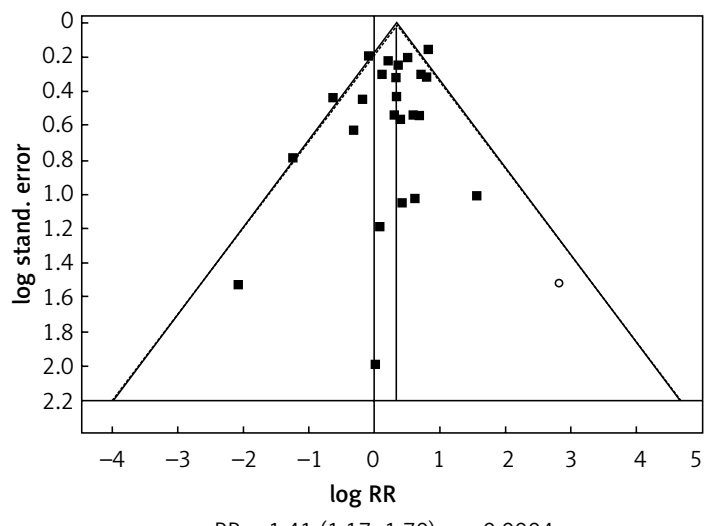

$\rightarrow-\mathrm{RR}=1.41(1.17 ; 1.70) p=0.0004$

$\mathrm{F}$

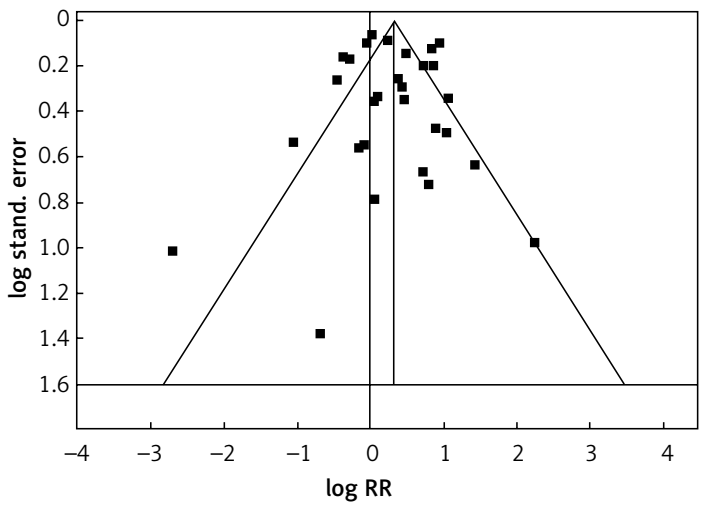

Figure 4. Publication bias of meta-analysis by Trim and Fill analysis. Funnel plots (RR - relative risk, random effect $95 \% \mathrm{Cl}$ ) show the distribution of published study outcomes (filled squares) vs. unpublished outcomes (open circles) estimated by Trim and Fill analysis. Dashed line represents RR and 95\% Cl with the added potentially unpublished studies and solid line represents published studies included in meta-analysis. Vertical dashed line represents the global estimate of safety. A - Blood and lymphatic disorders, B - anemia, C - gastrointestinal disorders, D - diarrhea, E - nausea, F - musculoskeletal and connective tissue disorders

and myalgia. Musculoskeletal adverse events are common ones related to therapy with prostanoid $\left(P \mathrm{PI}_{2}\right)$ or IP receptor agonists. Prostanoid-based therapy remains a critical component of optimal $\mathrm{PH}$ management, particularly for patients with the most severe disease. We can demonstrate a two- or three-fold increase in the risk of myalgia or jaw pain in cases where inhaled iloprost, treprostinil (both: p.o. and s.c.) or selexipag was added to the baseline. Current international guide- 
G

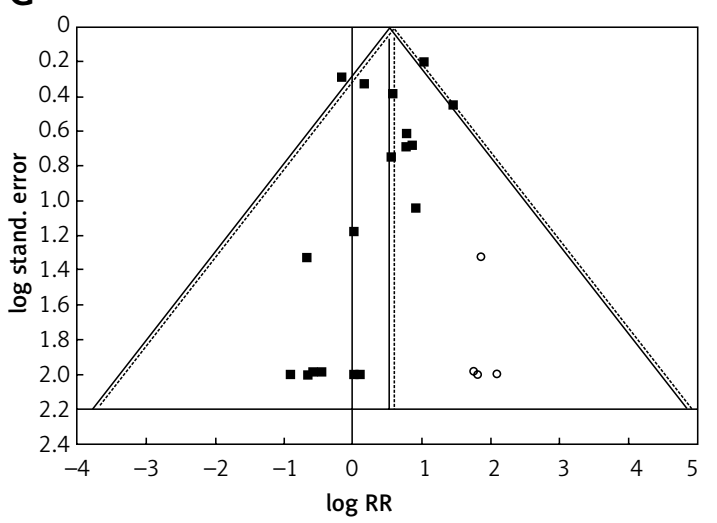

$\rightarrow \mathrm{RR}=1.73(1.32 ; 2.25) p<0.0001$

I

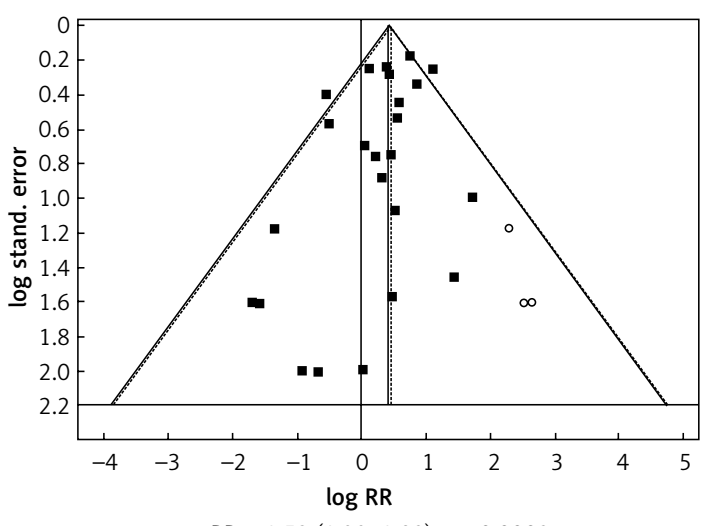

$\rightarrow-R R=1.53(1.22 ; 1.92) p=0.0002$

K

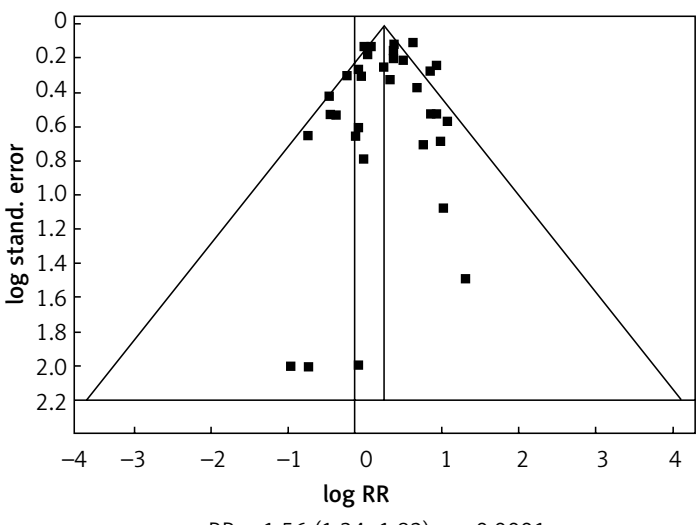

$\mathrm{H}$

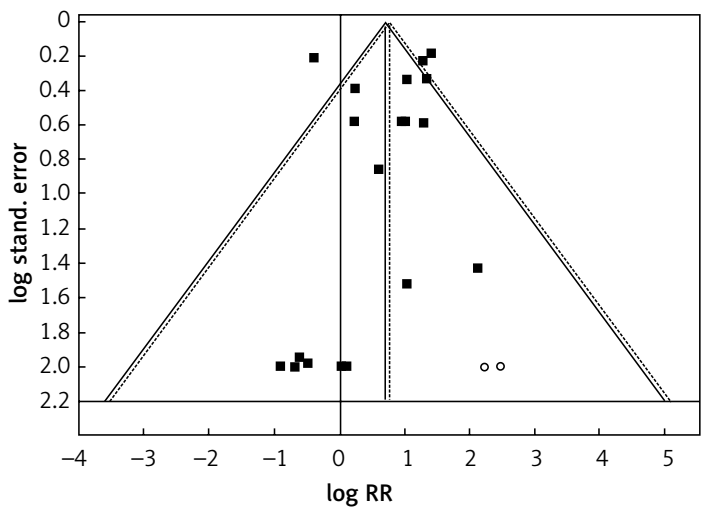

- $\mathrm{RR}=2.04(1.34 ; 3.11) p=0.0008$

$\ldots \mathrm{RR}=2.18(1.46 ; 3.26) p=0.0002$

J

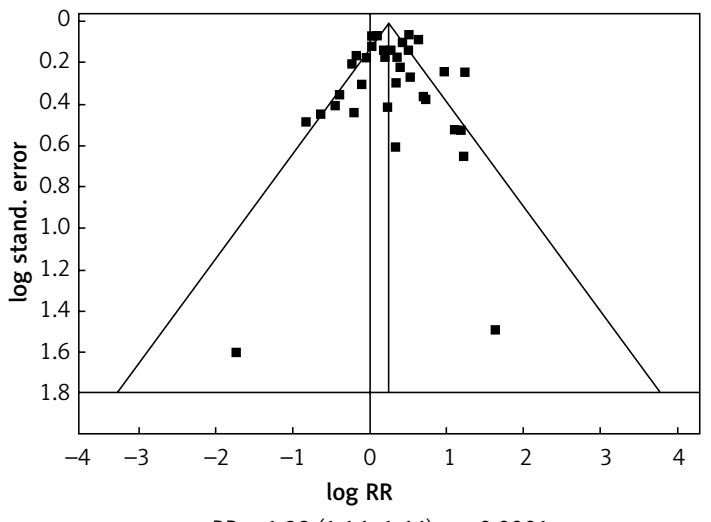

$R R=1.28(1.14 ; 1.44) p<0.0001$

L

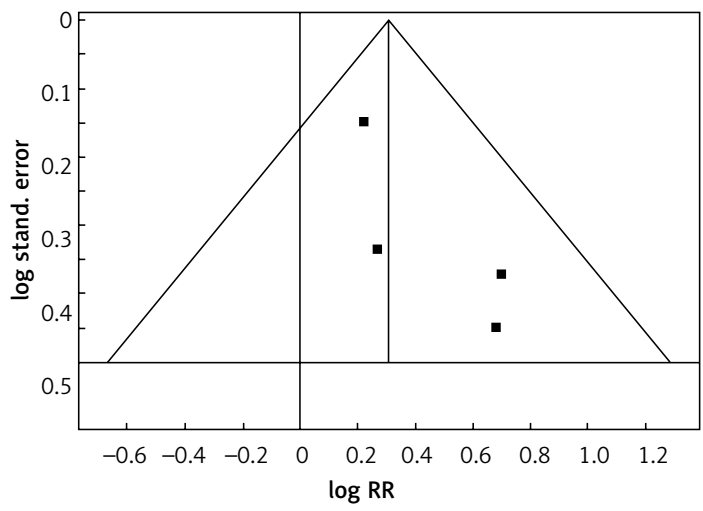

$\mathrm{RR}=1.36(1.07 ; 1.73) p=0.0108$

Figure 4. Cont. $\mathbf{G}$ - muscle pain, $\mathbf{H}$ - jaw pain, I - pain in limb, J - nervous system disorders, $\mathbf{K}$ - headache, $\mathbf{L}$ - respiratory and thoracic system disorders

lines recommend them both in non-vasoreactive and treatment-naive patients at high risk, with initial combination therapy including intravenous epoprostenol (class A, level I) or prostacyclin analogues. Selexipag is an orally administered IP receptor agonist very recently approved by the Food and Drug Administration (FDA) with indications for $\mathrm{PH}$ patients in World Health Organization functional class (WHO-FC) II and III (class B, level I) [15].
Many of the common side effects that were reported for prostanoids in single trials, e.g. flushing, headache, hypotension, nausea, vomiting and diarrhea, are generally dependent on the administered dose and they may disappear as treatment continues. Again, such reactions were reported for both parenteral and non-parenteral forms of treprostinil. Several symptoms can also arise as a consequence of vascular bed vasodilation by 
M
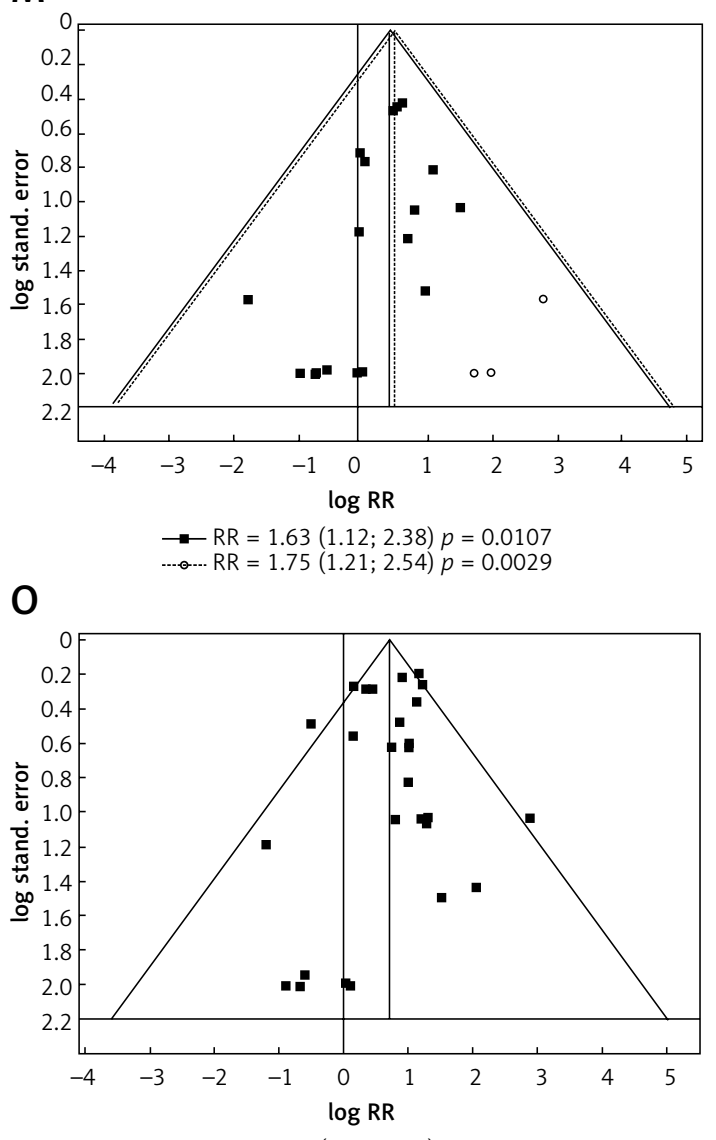

$R R=2.05(1.62 ; 2.60) p<0.0001$

Q

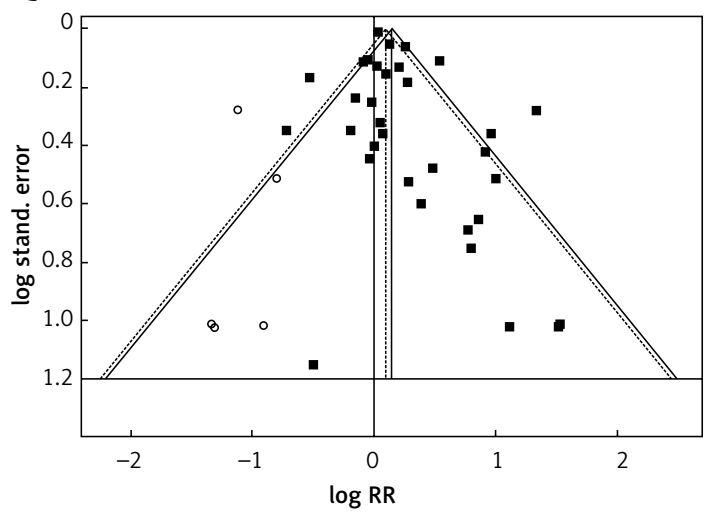

$\rightarrow-\mathrm{RR}=1.15(1.04 ; 1.28) p=0.0073$

$\mathrm{RR}=1.10(0.98 ; 1.22) p=0.0956$
$\mathrm{N}$

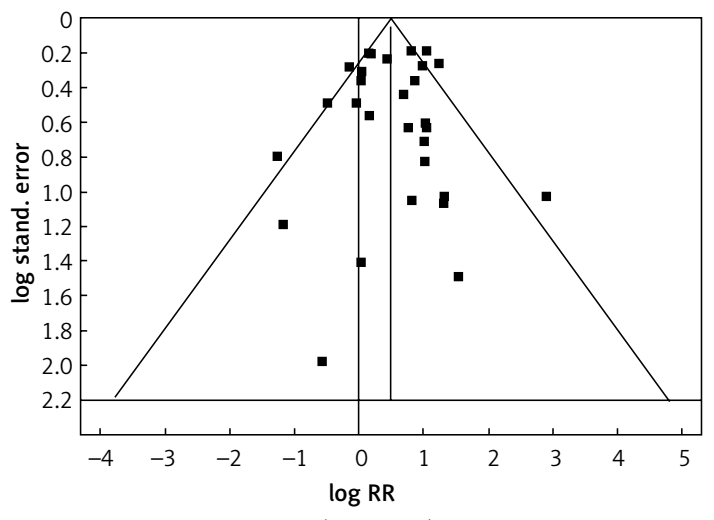

$\rightarrow \mathrm{RR}=1.63(1.30 ; 2.05) p<0.0001$

P

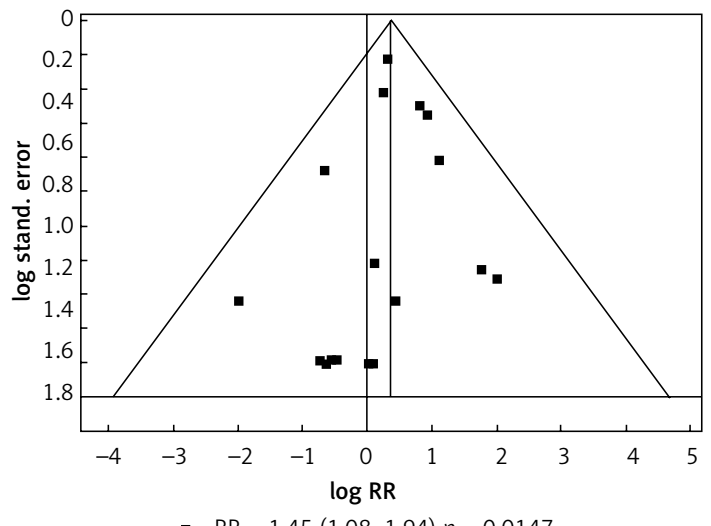

$\operatorname{RR}=1.45(1.08 ; 1.94) p=0.0147$

$\mathbf{R}$

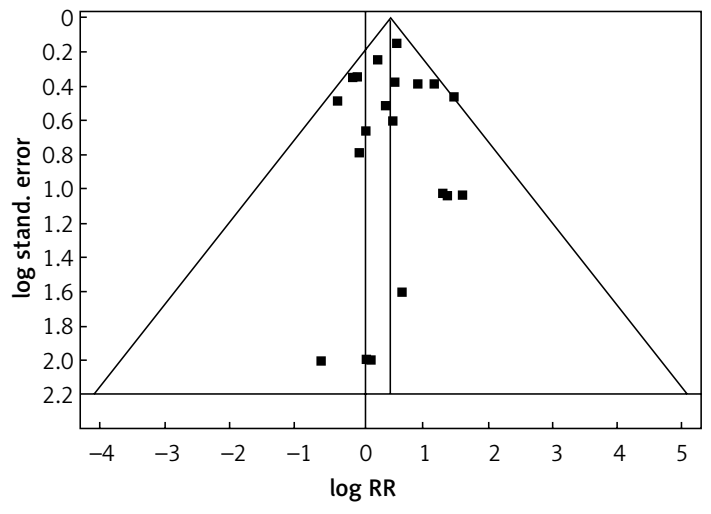

$\rightarrow \operatorname{RR}=1.44(1.21 ; 1.71) p=0.0004$

Figure 4. Cont. $\mathbf{M}$ - epistaxis, $\mathbf{N}$ - vascular disorders, $\mathbf{O}$ - flushing, $\mathbf{P}$ - eye disorders, $\mathbf{Q}$ - general disorders and administration site conditions, $\mathbf{R}$ - peripheral edema, $\mathbf{S}$ - discontinuations and $\mathbf{T}$ - SADRs. Non-significant result of Egger's test for A, C-G, I-P and R. According to visual inspection and results of Egger's test there is a suggestion of missing studies and publication bias excluding C, F, J, K, L, N, O, P, R

prostacyclin analogues and prostacyclin receptor agonists: the risk of events such as headache and flushing was increased by more than 1.5-fold. A two-fold increased risk for insomnia (treprostinil, p.o.) and 1.5-fold increased risk for cough (iloprost, inhaled) were also found. As demonstrated in a recent analysis by Leary et al. (2017) ADRs such as skin reactions, headaches and jaw pain were not associated with the mortality of patients receiving treprostinil (s.c.), while gastrointestinal side effects occurring during the first eight weeks following treprostinil infusion were associated with a $57 \%$ increase in the risk of mortality. The authors attribute this phenomena to poor nutri- 
S

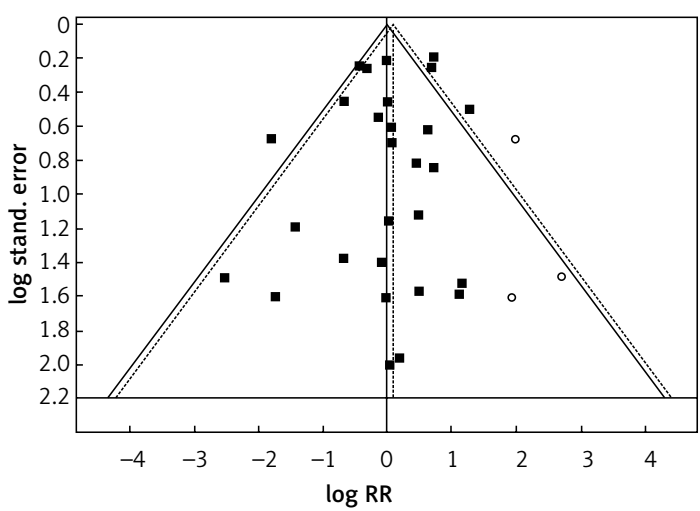

$\rightarrow R R=0.99(0.74 ; 1.32) p=0.9443$

$\ldots \rightarrow$ RR $=1.10(0.81 ; 1.48) p=0.5470$
$\mathrm{T}$

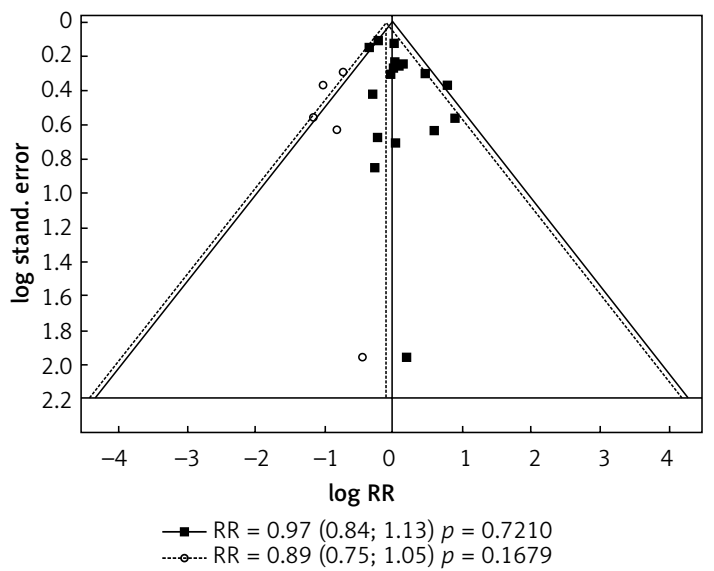

Figure 4. Cont. Result of Egger's test for $(\mathbf{S})$ and $(\mathbf{T})$ was non-significant. However, in the middle and right of the plot $(\mathbf{S})$, and in the top diagram $(\mathrm{T})$ there is a suggestion of missing studies, making publication bias plausible

tion caused by gastric events; when accompanied with low albumin and body mass index, this can worsen prognosis in $\mathrm{PH}$ patients [7].

Another target for $\mathrm{PH}$-specific agents is the endothelin pathway. ERAs play an essential role in the monotherapy of $\mathrm{PH}$ patients classified in WHO-FC II and III (class A, B-macitentan, level I), as well as being a component of both initial and sequential combined regimens [15]. The previous results from single clinical trials revealed that elevated liver aminotransferase values greater than three times normal occurred in $12.7 \%$ of patients receiving bosentan [16]. Twelve trials with an ERA therapeutic arm were included in the current metaanalysis, and this class of agents was found to be moderately safe. The most essential ERA-induced ADRs were classified among blood and lymphatic disorders with the subcategory anemia: iron deficiency is reported in $43 \%$ of patients with $\mathrm{iPAH}$. Such events may be associated with reduced exercise capacity and with higher mortality, independent of the presence or severity of anemia [17]. The relative risk of anemia was elevated by more than one and half times during therapy. It was independent of $\mathrm{PH}$-targeted therapy and it can be hypothesized that it was a consequence of clinical worsening.

Peripheral edema was demonstrated to be one of the most common adverse effects shared by prostanoid as well as ERA therapies in $\mathrm{PH}$. It was suggested that the fluid retention induced by ERAs might be a consequence of both the primary vascular and renal effects of endothelin receptor type A blockade [18]. However, in our analysis, such events were demonstrated to significantly increase, independently of therapeutic class; the final outcome seemed to be closely determined by agents targeting the endothelin pathway.

As previously reviewed, treatment-related adverse events with PDE-5i are generally mild to moderate, and consistent for this class of therapeutic agents; this is an important fact from a clinical point of view. Due to potential benefit of the investigated PDE-5 inhibitors in PH patients, such as significant pulmonary vasodilation, current European guidelines strongly recommend them for WHO-FC II and III patients (class A, level I) as monotherapy or in combination with other $\mathrm{PH}$-specific agents $[17,20]$. The most common adverse events for PDE-5 inhibitors can include headache (46\% vs. $39 \%$ placebo), flushing (12\% vs. $4 \%$ ), dyspepsia ( $12 \%$ vs. $7 \%)$, and back pain (12\% vs. $11 \%)$ [19, 20]. The observations from earlier single trials performed on patients with erectile dysfunction are confirmed by those of our present survey, based on patients with $\mathrm{PH}$. The risk of such events as headache or flushing was increased by more than one and a half times, and this seemed to be a consequence of the vasodilatory efficacy of sildenafil or tadalafil.

Eye disorders have been described previously as another adverse event specific to PDE-5 inhibitors and can involve such events as decrease in vision, flashes, bright colors, visual field defect, blurry vision, decrease in color vision or pain [21, 22]. In our survey, a substantial increase in relative risk of symptoms defined as 'eye disorders' was reported in cases where sildenafil but not tadalafil was added to the baseline. This phenomena can be explained by the 700-fold selectivity of tadalafil for PDE-5 over PDE-6.

Out of 36 trials that were included in the analysis, the participants of 18 received at least two $\mathrm{PH}$-specific therapies. As we mentioned above, the combination regimens did not increase statistically significantly relative risk for severe ADRs. Conversely, other than severe ADRs were more pronounced in patients receiving the combined regimens. In relation to the particular events according to the MedRA dictionary, a significant 
Table II. Summary of sensitivity analysis. The analysis was performed to exclude potential studies with the biggest RR outlier. In cases where any single study did not influence the statistical significance of the final outcome, all trials were included in the analysis. ID - MedRA codes: categories with selected subcategories

\begin{tabular}{|c|c|c|}
\hline Category & ID & Studies removed \\
\hline Blood and lymphatic system disorders & 10005329 & All studies included \\
\hline Anemia & 10002034 & All studies included \\
\hline Cardiac disorders & 10007541 & All studies included \\
\hline Palpitation & 10033556 & All studies included \\
\hline Right ventricular failure & 10039163 & All studies included \\
\hline Congenital, familial and genetic disorders & 10010331 & All studies included \\
\hline Ear and labyrinth disorders & 10013993 & All studies included \\
\hline Endocrine disorders & 10014698 & All studies included \\
\hline Eye disorders & 10015919 & All studies included \\
\hline Gastrointestinal disorders & 10017947 & All studies included \\
\hline Diarrhea & 10012735 & All studies included \\
\hline Nausea & 10028813 & All studies included \\
\hline Vomiting & 10047700 & All studies included \\
\hline General disorders and administration site conditions & 10018065 & All studies included \\
\hline Fatigue & 10016256 & All studies included \\
\hline Peripheral edema & 10034570 & 2 studies removed $[38,43]$ \\
\hline Hepatobiliary disorders & 10019805 & All studies included \\
\hline Immune system disorders & 10021428 & All studies included \\
\hline Infections and infestations & 10021881 & All studies included \\
\hline Upper respiratory tract infections & 10046309 & 1 study removed [38] \\
\hline Injury, poisoning and procedural complications & 10022117 & All studies included \\
\hline Investigations & 10022891 & 1 study removed [31] \\
\hline Metabolism and nutrition disorders & 10027433 & All studies included \\
\hline Musculoskeletal and connective tissue disorders & 10028395 & 1 study removed [31] \\
\hline Arthralgia & 10003246 & All studies included \\
\hline Jaw pain & 10023157 & All studies included \\
\hline Muscle pain & 10028322 & All studies included \\
\hline Pain in limb & 10033447 & All studies included \\
\hline Neoplasms benign, malignant and unspecified (incl. cysts and polyps) & 10029104 & All studies included \\
\hline Nervous system disorders & 10029205 & All studies included \\
\hline Headache & 10019211 & All studies included \\
\hline Dizziness & 10013573 & All studies included \\
\hline Syncope & 10042772 & All studies included \\
\hline Pregnancy, puerperium and perinatal conditions & 10036585 & All studies included \\
\hline Psychiatric disorders & 10037175 & All studies included \\
\hline Renal and urinary disorders & 10038359 & All studies included \\
\hline
\end{tabular}


Table II. Cont.

\begin{tabular}{|lcc|}
\hline Category & ID & Studies removed \\
\hline Reproductive system and breast disorders & 10038604 & All studies included \\
\hline Respiratory, thoracic and mediastinal disorders & 10038738 & 2 studies removed [56, 57] \\
\hline Cough & 10011224 & 1 study removed [31] \\
\hline Dyspnea & 10013968 & All studies included \\
\hline Ekistaxis & 10015090 & 1 study removed [36] \\
\hline Rash & 10040785 & 3 studies removed \\
\hline Social circumstances & 10037844 & 1 study removed [24] \\
\hline Surgical and medical procedures & 10041244 & All studies included \\
\hline Vascular disorders & 10042613 & All studies included \\
\hline Flushing & 10047065 & All studies included \\
\hline
\end{tabular}

tendency toward increased RR was observed for such ADRs as blood and lymphatic system disorders with anemia subgroup, gastrointestinal disorders with diarrhea and nausea subgroups, respiratory and thoracic diseases, nervous system disorders with headache, vascular events, myalgia and pain in limb. The first four main categories of ADRs tended to occur more often in the case of administration of at least two drugs as compared to monotherapy.

Our analysis has several limitations. First, it was not possible to obtain individual patient-level data from included trials, and this fact can weak en the accuracy of the obtained results. Second, of the 36 trials, only four concerned inhalation, two subcutaneous and one intravenous administration, which may have exaggerated the input of oral treatments on the results of safety. The third limitation concerned the variable reporting of adverse events (not all categories were included in all studies) and the different duration of trials, which could affect the observations of the safety of administered agents. Fourth, the definitions of severe ADRs were varied and commonly not provided in particular trials included in the meta-analysis. Fifth, due to the significant impact on the final outcome, some trials were removed during sensitivity analysis. This concerned only a few ADR categories. Similarly, our funnel plot analysis showed a graphic and statistical asymmetry for only a few ADRs. Nevertheless, publication bias favoring the publication of positive results (better safety profile) in such cases is possible. In addition, some of the reported side effects, such as dyspnea, fatigue, edema, anemia or cardiac disorders, may occur in response to underlying pulmonary hypertension. In this case, the interpretation of the ADRs associated with specific $\mathrm{PH}$ therapies can be inaccurate.

In conclusion, the applied therapies were associated with a non-significant risk of ADRs in more than half of their main categories according to MedRA. Conversely, the overall frequency for events classified into remaining categories (e.g. blood and lymphatic system, the gastrointestinal, musculoskeletal or nervous systems, or the respiratory or vascular disorders) were confirmed to be more than $1 / 10$ or $1 / 100$. Such ADRs as blood and lymphatic system disorders with anemia subgroup, gastrointestinal disorders with diarrhea and nausea subgroups, respiratory and thoracic diseases or nervous system disorders with headache were identified to occur more often in combination regimens as compared to monotherapy. Their risk can be increased when agents targeting prostacyclin pathway are used, especially. The risk of cessation was comparable between patients receiving $\mathrm{PH}$-specific agents in monotherapy or combination, with the combined regimen demonstrating only a slightly greater risk.

\section{Acknowledgements}

The study was supported by the Medical University of Lodz, grant no. 503/3-011-02/503-31002.

\section{Conflict of interest}

The authors declare no conflict of interest.

\section{References}

1. Escribano-Subias P, Blanco I, López-Meseguer M, et al. REHAP investigators. Survival in pulmonary hyperten- 
sion in Spain: insights from the Spanish registry. Eur Respir J 2012; 40: 596-603.

2. Humbert M. Pulmonary arterial hypertension and chronic thromboembolic pulmonary hypertension: pathophysiology. Eur Respir Rev 2010; 19: 59-63.

3. Lee WT, Ling Y, Sheares KK, Pepke-Zaba J, Peacock AJ, Johnson MK. Predicting survival in pulmonary arterial hypertension in the UK. Eur Respir J 2012; 40: 604-11.

4. Ling Y, Johnson MK, Kiely DG, et al. Changing demographics, epidemiology, and survival of incident pulmonary arterial hypertension: results from the pulmonary hypertension registry of the United Kingdom and Ireland. Am J Respir Crit Care Med 2012; 186: 790-6.

5. Pullamsetti SS, Schermuly R, Ghofrani A, Weissmann N, Grimminger F, Seeger W. Novel and emerging therapies for pulmonary hypertension. Am J Respir Crit Care Med 2014; 189: 394-400.

6. Lajoie AC, Lauzière G, Lega JC, et al. Combination therapy versus monotherapy for pulmonary arterial hypertension: a meta-analysis. Lancet Respir Med 2016; 4: 291-305.

7. Leary PJ, Kang S, Kolb TM, et al. What's in a side effect? The association between pulmonary vasodilator adverse drug events and clinical outcomes in patients with pulmonary arterial hypertension. Int J Cardiol 2017; 240: 386-91.

8. Liberati A, Altman DG, Tetzlaff J, et al. The PRISMA statement for reporting systematic reviews and meta-analyses of studies that evaluate health care interventions: explanation and elaboration. PLoS Med 2009; 6: e1000100.

9. Jadad AR, Moore RA, Carroll D, et al. Assessing the quality of reports of randomized clinical trials: is blinding necessary? Control Clin Trials 1996; 17: 1-12.

10. DerSimonian R, Laird N. Meta-analysis in clinical trials Control Clin Trials 1986; 7: 177-88.

11. Begg CB, Mazumdar M. Operating characteristics of a rank correlation test for publication bias. Biometrics 1994; 50: 1088-101.

12. Egger M, Davey Smith G, Schneider M, Minder C. Bias in meta-analysis detected by a simple, graphical test. BMJ 1997; 315: 629-34.

13. Duval S, Tweedie R. Trim and fill: A simple funnel-plotbased method of testing and adjusting for publication bias in meta-analysis. Biometrics 2000; 56: 455-63.

14. Galiè N, Palazzini M, Manes A. Pulmonary arterial hypertension: from the kingdom of the near-dead to multiple clinical trial meta-analyses. Eur Heart 2010; 31: 2080-6.

15. Galiè N, Humbert M, Vachiery JL, et al. 2015 ESC/ERS Guidelines for the diagnosis and treatment of pulmonary hypertension: The Joint Task Force for the Diagnosis and Treatment of Pulmonary Hypertension of the European Society of Cardiology (ESC) and the European Respiratory Society (ERS): Endorsed by: Association for European Paediatric and Congenital Cardiology (AEPC), International Society for Heart and Lung Transplantation (ISHLT). Eur Respir J 2015; 46: 903-75.

16. Frumkin LR. The pharmacological treatment of pulmonary arterial hypertension. Pharmacol Rev 2012; 64 : 583-620.

17. Ruiter $\mathrm{G}$, Lankhorst $\mathrm{S}$, Boonstra A, et al. Iron deficiency is common in idiopathic pulmonary arterial hypertension Eur Respir J 2011; 37: 1386-91.

18. Shapiro S, Pollock DM, Gillies H, et al. Frequency of ede$\mathrm{ma}$ in patients with pulmonary arterial hypertension receiving ambrisentan. Am J Cardiol 2012; 110: 1373-7.

19. Gresser U, Gleiter $\mathrm{CH}$. Erectile dysfunction: comparison of efficacy and side effects of the PDE-5 inhibitors silde- nafil, vardenafil and tadalafil - review of the literature. Eur J Med Res 2002; 7: 435-46.

20. Brock GB, McMahon CG, Chen KK, et al. Efficacy and safety of tadalafil for the treatment of erectile dysfunction: results of integrated analyses. J Urol 2002; 168: 1332-6.

21. Hellstrom WJ, Gittelman M, Karlin G, et al.; Vardenafil Study Group. Sustained efficacy and tolerability of vardenafil, a highly potent selective phosphodiesterase type 5 inhibitor, in men with erectile dysfunction: results of a randomized, double-blind, 26-week placebo-controlled pivotal trial. Urology 2003; 61: 8-14.

22. Laties AM. Vision disorders and phosphodiesterase type 5 inhibitors: a review of the evidence to date. Drug Saf 2009; 32: 1-18.

23. Olschewski H, Simonneau G, Galiè N, et al.; Aerosolized Iloprost Randomized Study Group. Inhaled iloprost for severe pulmonary hypertension. N Engl J Med 2002; 347: 322-9.

24. Galiè N, Barberà JA, Frost AE, et al. Initial use of ambrisentan plus tadalafil in pulmonary arterial hypertension. N Engl J Med 2015; 373: 834-44.

25. Galiè N, Olschewski H, Oudiz R, et al. Ambrisentan for the treatment of pulmonary arterial hypertension: results of the ambrisentan in pulmonary arterial hypertension, randomized, double-blind, placebo-controlled, multicenter, efficacy (ARIES) study 1 and 2. Circulation 2008; 117: 3010-9.

26. ClinicalTrials.gov. ARTEMIS-PH - Study of Ambrisentan in Subjects With Pulmonary Hypertension Associated With Idiopathic Pulmonary Fibrosis (ARTEMIS-PH). https://clinicaltrials.gov/ct2/show/NCT00879229 (access: 26 May 2017).

27. Badesch D, Bodin F, Channick R, et al. Complete results of the first randomized, placebo-controlled study of bosentan, a dual endothelin receptor antagonist, in pulmonary arterial hypertension. Curr Ther Res Clin 2002; 63: 227-46.

28. Rubin L, Badesch D, Barst R, et al. Bosentan therapy for pulmonary arterial hypertension. N Engl J Med 2002; 346: 896-903.

29. Humbert M, Barst RJ, Robbins IM, et al. Combination of bosentan with epoprostenol in pulmonary arterial hypertension: BREATHE-2. Eur Respir J 2004; 24: 353-9.

30. Galiè N, Beghetti $M$, Gatzoulis $M$, et al.; Bosentan Randomized Trial of Endothelin Antagonist Therapy-5 (BREATHE-5) Investigators. Bosentan therapy in patients with Eisenmenger syndrome: a multicenter, double-blind, randomized, placebo-controlled study. Circulation 2006; 114: 48-54.

31. McLaughlin V, Channick RN, Ghofrani HA, et al. Bosentan added to sildenafil therapy in patients with pulmonary arterial hypertension. Eur Respir J 2015; 46: 405-13.

32. Denton C, Humbert M, Rubin L, Black M. Bosentan treatment for pulmonary arterial hypertension related to connective tissue disease: a subgroup analysis of the pivotal clinical trials and their open-label extensions. Ann Rheum Dis 2006; 65: 1336-40.

33. Bonderman D, Pretsch I, Steringer-Mascherbauer R, et al. Acute hemodynamic effects of riociguat in patients with pulmonary hypertension associated with diastolic heart failure (DILATE-1): a randomized, double-blind, placebo-controlled, single-dose study. Chest 2014; 146: 1274-85.

34. Galiè N, Rubin LJ, Hoeper M, et al. Treatment of patients with mildly symptomatic pulmonary arterial hypertension with bosentan (EARLY study): a double-blind, randomised controlled trial. Lancet 2008; 371: 2093-100. 
35. Jing ZC, Parikh K, Pulido T, et al. Efficacy and safety of oral treprostinil monotherapy for the treatment of pulmonary arterial hypertension: a randomized, controlled trial. Circulation 2013; 127: 624-33.

36. Tapson VF, Torres F, Kermeen F, et al. Oral treprostinil for the treatment of pulmonary arterial hypertension in patients on background endothelin receptor antagonist and/or phosphodiesterase type 5 inhibitor therapy (the FREEDOM-C study): a randomized controlled trial. Chest 2012; 142: 1383-90

37. Tapson VF, Jing ZC, Xu KF, et al. Oral treprostinil for the treatment of pulmonary arterial hypertension in patients receiving background endothelin receptor antagonist and phosphodiesterase type 5 inhibitor therapy (the FREEDOM-C2 study): a randomized controlled trial. Chest 2013; 144: 952-8.

38. Sitbon O, Channick R, Chin KM, et al. Selexipag for the treatment of pulmonary arterial hypertension. $\mathrm{N}$ Engl J Med 2015; 37: 2522-33

39. Iversen K1, Jensen AS, Jensen TV, Vejlstrup NG, Søndergaard L. Combination therapy with bosentan and sildenafil in Eisenmenger syndrome: a randomized, placebo-controlled, double-blinded trial. Eur Heart J 2010; 31: 1124-31.

40. Machado RF, Barst R, Yovetich N, et al. Hospitalization for pain in patients with sickle cell disease treated with sildenafil for elevated TRV and low exercise capacity. Blood 2011; 118: 855-64.

41. Simonneau G, Rubin LJ, Galiè N, et al. Addition of sildenafil to long-term intravenous epoprostenol therapy in patients with pulmonary arterial hypertension: a randomized trial. Ann Intern Med 2008; 149: 521-30.

42. Galiè N, Müller K, Scalise AV, Grünig E. PATENT PLUS: a blinded, randomised and extension study of riociguat plus sildenafil in PAH. Eur Respir J 2015; 45: 1314-22.

43. Ghofrani HA, Galiè N, Grimminger F, et al. Riociguat for the treatment of pulmonary arterial hypertension. N Engl J Med 2013; 36: 330-40.

44. ClinicalTrials.gov Assess the Efficacy and Safety of Sildenafil When Added to Bosentan in the Treatment of Pulmonary Arterial Hypertension. https://clinicaltrials.gov/ ct2/show/study/NCT00323297 (access: 29 May 2017).

45. Barst RJ, Oudiz RJ, Beardsworth A, et al. Tadalafil monotherapy and as add-on to background bosentan in patients with pulmonary arterial hypertension. J Heart Lung Transplant 2011; 30: 632-43.

46. Rubenfire M, McLaughlin V, Allen R, et al. Transition from IV epoprostenol to subcutaneous treprostinil in pulmonary arterial hypertension: a controlled trial. Chest 2007; 132: 757-63.

47. Sastry B, Narasimhan C, Reddy N, Raju B. Clinical efficacy of sildenafil in primary pulmonary hypertension. A randomized, placebo-controlled, double-blind, crossover study. J Am Coll Cardiol 2004; 43: 1149-53.

48. Pulido T, Adzerikho I, Channick RN, et al. Macitentan and morbidity and mortality in pulmonary arterial hypertension. N Engl J Med 2013; 369: 809-18.

49. Simonneau G, Barst R, Galie N, et al. Continuous subcutaneous infusion of treprostinil, a prostacyclin analogue, in patients with pulmonary arterial hypertension: a double-blind, randomized, placebo-controlled trial. Am J Respir Crit Care Med 2002; 165: 800-4.

50. Simonneau G, Torbicki A, Hoeper MM, et al. Selexipag: an oral, selective prostacyclin receptor agonist for the treatment of pulmonary arterial hypertension. Eur Respir J 2012; 40: 874-80.
51. McLaughlin VV, Oudiz RJ, Frost A, et al. Randomized study of adding inhaled iloprost to existing bosentan in pulmonary arterial hypertension. Am J Respir Crit Care Med 2006; 174: 1257-63.

52. Wirostko B, Tressler C, Hwang L, Burgess G, Laties A. Ocular safety of sildenafil citrate when administered chronically for pulmonary arterial hypertension: results from phase III, randomised, double masked, placebo controlled trial and open label extension. BMJ 2012; 344: e554.

53. Galiè N, Ghofrani H, Torbicki A, et al.; Sildenafil Use in Pulmonary Arterial Hypertension (SUPER) Study Group. Sildenafil citrate therapy for pulmonary arterial hypertension. N Engl J Med 2005; 353: 2148-57.

54. McLaughlin VV, Benza RL, Rubin LJ, et al. Addition of inhaled treprostinil to oral therapy for pulmonary arterial hypertension: a randomized controlled clinical trial. J Am Coll Cardiol 2010; 55: 1915-22.

55. Hiremath J, Thanikachalam S, Parikh K, et al. Exercise improvement and plasma biomarker changes with intravenous treprostinil therapy for pulmonary arterial hypertension: a placebo-controlled trial. J Heart Lung Transplant 2010; 29: 137-49.

56. ClinicalTrials.gov The "VISION" Trial: Ventavis Inhalation With Sildenafil to Improve and Optimize Pulmonary Arterial Hypertension (VISION). https://clinicaltrials.gov/ ct2/show/NCT00302211 (access: 29 May 2017).

57. Zhuang Y, Jiang B, Gao H, Zhao W. Randomized study of adding tadalafil to existing ambrisentan in pulmonary arterial hypertension. Hypertens Res 2014; 37: 507-12. 